\title{
The Biofilm Model of Freter: a review
}

\author{
Mary Ballyk ${ }^{1}$, Don Jones ${ }^{2}$, and Hal Smith ${ }^{3}$ \\ 1 Department of Mathematical Sciences, New Mexico State University, Las \\ Cruces, NM 88003-8001 mballyk@nmsu.edu \\ 2 Department of Mathematics and Statistics, Arizona State University, Tempe, AZ \\ 85287-1804 dajones@math. asu.edu \\ 3 Supported by NSF Grant DMS 0414270, Department of Mathematics and \\ Statistics, Arizona State University, Tempe, AZ 85287-1804 halsmith@asu.edu
}

Summary. R. Freter et al (1983) developed a simple chemostat-based model of competition between two bacterial strains, one of which is capable of wall-growth, in order to illuminate the role of bacterial wall attachment on the phenomenon of colonization resistance in the mammalian gut. Together with various collaborators, we have re-formulated the model in the setting of a tubular flow reactor, extended the interpretation of the model as a biofilm model, and provided both mathematical analysis and numerical simulations of solution behavior. The present paper provides a review of the work in $[5,6,4,52,50,36,33,32,35,34]$.

Introduction. The ability of bacteria to colonize surfaces forming biofilms and thereby to create a refuge from the vagaries of fluid advection has stimulated a great deal of recent research in a variety of disciplines. The importance of wall growth was first made apparent to us from the work of microbiologist Rolf Freter and his colleagues [25, 27, 26, 24]. Their mathematical models of the phenomenon of colonization resistance in the mammalian gut showed that bacterial wall attachment could play a crucial role in the observed stability of the natural microflora of the gut to invasion by non-indigenous microorganisms. The authors had the pleasure of learning of this work first hand from a lecture by Freter at the Microbial Ecology Workshop organized by Frank Hoppensteadt and Smith at Arizona State University in 1997. Our own work can be traced to a collaboration that began at this workshop.

While Freter's model was formulated in a CSTR (chemostat) setting which is natural since this reactor mimics the mouse cecum, the animal model for gut research, we felt that another natural setting was the plug flow reactor (PFR) where bacterial motility, fluid advection, and other spatial effects could play a larger role. The flow reactor more accurately reflects the environment of the large intestine of humans. Thus, Ballyk and Smith formulated a family of models for microbial growth and competition for limiting substrate and wall-attachment sites in our first work [5]. This paper focused on describing the model equations which consist of a system of parabolic partial differential 
equations for a diffusing substrate and for randomly motile bacteria in the fluid environment of a thin (one-dimensional) tube coupled to a system of ordinary differential equations for the immobile, wall-attached bacteria growing on the tubular surface. It was natural to begin a mathematical analysis of the model by considering only a single bacterial strain in the reactor in [6].

The phenomena of colonization resistance in the gut remained the primary motivation for our early work in $[5,6,4,52,50]$. However, we quickly became aware of the rapidly developing literature on biofilms and began to view our models in this more general context. Our subsequent work [36, 33, 32, 35, $34,30]$ was largely motivated by our view that the Freter model is a crude model of a biofilm. A biofilm is simply a layer of material coating a surface, usually immersed in a fluid environment, made up of bacteria and an extracellular matrix exuded by the bacteria which provides an environment for growth. From the bacterial point of view, a biofilm is a comfortable refuge. Examples of a biofilm include the scum that grows on a rock in a stream, dental plaque on teeth, the surface slime that forms on the inside surface of water pipes, and a similar coating of the surface of the large intestine of mammals. These bacterial layers can have serious negative consequences in many man made environments. They contaminate medical devices such as contact lenses, implants, catheters, and stints; they can contaminate food and medicinal production facilities and air-conditioning and water circulation systems. Biofilms are notoriously difficult to eradicate once established.

The study of biofilms has exploded in the last two decades, largely driven by recent advances in noninvasive microscopy, staining techniques, and genetic probes. Contributing to this explosion of interest was the realization that many human disease processes are essentially biofilm infections of organs, e.g., Periodontitus in teeth and gums, Otitus media in the ear, and Cystic fibrosis pneumonia in the lung, to name only a few [28]. In ways not yet completely understood, these biofilm infections are difficult to treat using antibiotics [53]. It is hoped that increased understanding of the biofilm environment will lead to improved modes of therapy for these diseases.

From this recent attention focused on biofilms has come the realization that what scientists had learned about bacteria, based on more than a century of studying them in suspension as isolated cells, e.g. in a chemostat, or in simplified batch culture environments, did not prepare them for the observed level of complexity these organisms display in the biofilm setting. It is now recognized that almost all bacteria live in biofilm communities of remarkable structure which are attached to surfaces and in which individual bacteria are capable of expressing whole suites of genes not previously known that allow them to communicate and coordinate their activities. Quorum sensing, a bacterial communication mechanism based on the exchange of an internally produced small molecule and the detection of its extracellular concentration, has been shown to control aspects of biofilm formation and development and, in cases of biofilm infections of tissue, to control the expression of genes controlling virulence factors. For example, it is advantageous for bacteria not to 
express virulence until a suitable defence, a mature biofilm environment, has been established. The reader interested in more background information concerning biofilms should consult the many review articles of Costerton et al. $[17,16,18,19]$ and the monograph of Bryers [11]. For an update on current directions in the study of biofilms consult the special issue (January 2005) of the journal Trends in Microbiology devoted to biofilm research.

Along with this increase in the interest in biofilms there has been increasing interest in mathematical models of biofilms. Below we give a necessarily limited review of some of this literature. A classical but now somewhat dated reference is the volume "Biofilms" edited by Characklis and Marshall [12] where partial differential equations are used to model steady state (time independent) bacterial densities. An updated edited volume "Biofilms II" [11] indicates some recent directions of research. Most recent modeling in the field has been directed towards understanding the mechanisms underlying the remarkable variety of spatial structure observed in different biofilms. These structures can be as simple as flat layers with little or no structure to fields of mushroom-like structures protruding from the surface. Modeling the evolution of a biofilm in detail requires dealing with the physical forces giving rise to fluid motion, advection and diffusion of nutrients and wastes, the forces acting on the moving interface between the biofilm and bulk fluid on the one hand and also including the biological aspects including growth, production of extra-cellular matrix, and attachment and detachment of cells and matrix from the biofilm. Classically, these considerations lead naturally to large systems of partial differential equations. Recent papers of Cogan and Keener [14], Eberl et al. [23], Dockery and Klapper [22], examine various aspects of biofilm morphology using continuum models that lead to systems of partial differential equations with a moving biofilm-fluid interface for the growing biofilm. Cellular automaton models and related Individual-based models have recently been used by Wimpenny and Colasanti [57], Kreft et al. [38], and Laspidou and Rittmann [40] to model biofilm development. Hybrid models employing a mixture of continuum modeling and cellular automata have been employed by Picioreanu et al. [44] and Noguera et al. [42]. Dillon et al. [21] combine partial differential equations for fluid flow, chemical densities with individual-based modeling of cells moving in response to forces to model biofilm formation. An up to date review of biofilm modeling by Noguera et al. [41] is useful. With the exception of $[22,14]$, most of these models are so complex that they can be investigated only using sophisticated numerical simulations.

There are few simple, conceptual biofilm models which are amenable to mathematical analysis yet which yield significant and useful results. Simple models do not attempt to provide much detail on the spatial structure of biofilms but aim to provide information on conditions suitable for biofilm establishment and maintenance and which model the formation of biofilms directly, starting from an inoculum of planktonic bacteria. Among the more widely known of these, we mention Topiwala and Hamer [55], Baltzis and Fredrickson [7], Bakke et al. [8], and Pilyugin and Waltman [45]. Less known 
is the work of R. Freter and his group (Freter [25, 24] and Freter et al. [26, 27]), who formulated a mathematical model to understand the phenomena of colonization resistance in the mammalian gut (stability of resident microflora to colonization). Essentially, their model can be viewed as a crude biofilm model. In contrast to state of the art biofilm models cited above, the Freter model completely ignores the three-dimensional spatial structure of the biofilm.

Our view is that the Freter model provides a useful yet mathematically tractable model of a biofilm which focuses primarily on the bacterial interactions. The present chapter provides an overview of our previous work in $[5,6,4,52,50,36,33,32,35,34]$. More recent work on gene transfer in biofilms [30,31], based on the biofilm models considered here is reviewed in [31]. The authors wish to acknowledge collaborators L. Dung, H. Kojouharov, E. Stemmons, X.-Q. Zhao who have contributed substantially towards portions of the work presented here.

As our models use nonlinear partial differential equations to capture random motility of cell populations and diffusion of nutrients, we will employ some of the same techniques used in Chapters 3 and 4 where spatial movement also plays an important role.

\section{The Freter Model}

The Freter model describes a microbial population in a fluid environment with a bounding surface, a portion of which may be colonized by the bacteria forming a biofilm. At any given moment of time, the bacterial population can be viewed as consisting of cells suspended in the fluid, usually called planktonic cells, and those adhering to the surface, called adherent cells. Planktonic cells can adhere to the biofilm becoming adherent cells and adherent cells may detach from the biofilm becoming planktonic cells. The Freter model is based on the following assumptions:

1. there are a finite number of available colonization sites on the wall and thus a maximum attainable areal density of adherent bacteria.

2. planktonic bacteria are attracted to the wall at a rate proportional to planktonic cell density and the fraction of unoccupied colonization sites on the wall.

3. adherent cells are sloughed off into the fluid at a rate proportional to their density.

4. daughter cells of adherent bacteria compete for space on the wall: A fraction $G$ of the daughter cells find attachment sites and the fraction $1-G$ do not and are forced into the fluid. $G$ is a decreasing function of wall occupancy.

Model parameters are described in the Table 1.

If $w$ denotes the areal density of wall-adherent bacteria and $w_{\max }$ the maximum attainable density, then the wall occupancy is given by 


\begin{tabular}{lll}
\hline Symbol & Description & Dimension \\
\hline$u$ & biomass concentration of planktonic bacteria. & $m l^{-3}$ \\
$w$ & areal biomass density of adherent bacteria. & $\mathrm{ml}^{-2}$ \\
$w_{\max }$ & maximum areal biomass density of adherent bacteria. & $\mathrm{ml}^{-2}$ \\
$W=w / w_{\max }$ & wall occupancy fraction. & - \\
$G(W)$ & fraction of daughter cells that find wall sites. & - \\
$\beta$ & sloughing rate of adherent bacteria. & $t^{-1}$ \\
$\alpha$ & rate constant of adhesion. & $t^{-1}$ \\
$S$ & concentration of limiting substrate. & $\mathrm{ml}^{-3}$ \\
$S_{0}$ & concentration of the substrate in the feed. & $m l^{-3}$ \\
$\gamma$ & yield constant. & - \\
$f_{u}(S)$ & specific growth rate of planktonic bacteria. & $t^{-1}$ \\
$f_{w}(S)$ & specific growth rate of adherent bacteria. & $t^{-1}$ \\
$k$ & planktonic cell death rate. & $t^{-1}$ \\
$k_{w}$ & adherent cell death rate. & $t^{-1}$ \\
\hline
\end{tabular}

Table 1. Model parameters and functions

$$
W=w / w_{\max }
$$

Planktonic cells, with density $u$, are attracted to the wall at rate $\alpha u(1-W)$, proportional to their density and to the unoccupied fraction $1-W$ of wall sites (see also Baltzis and Fredrickson [7]). It is reasonable to assume that $G=G(W)$ is a decreasing function of the occupation fraction $W$ because a more fully saturated wall provides less chance for a daughter cell to find space on it. Freter [25] employs the rational function

$$
G(W)=\frac{1-W}{1.1-W}
$$

Bacteria consume substrate $S$ at (per unit biomass) rate

$$
\begin{aligned}
f_{u}(S) & =\frac{m_{u} S}{a_{u}+S}, \text { planktonic cells, } \\
f_{w}(S) & =\frac{m_{w} S}{a_{w}+S}, \text { adherent cells, }
\end{aligned}
$$

with conversion to biomass with yield constant $\gamma$. Cell death rates are introduced for later use when anti-microbials are considered.

\section{The Chemostat-Based Model}

The Freter model was originally proposed in the setting of a CSTR of volume $V$, colonizable surface area $A$ and flow rate $F$ (Freter [25], Freter at al. [26]) where it takes the form $(D=F / V, \delta=A / V)$ : 


$$
\begin{aligned}
S^{\prime} & =D\left(S^{0}-S\right)-\gamma^{-1}\left[u f_{u}(S)+\delta w f_{w}(S)\right] \\
u^{\prime} & =u\left[f_{u}(S)-D-k\right]+\beta \delta w+\delta w f_{w}(S)[1-G(W)]-\alpha u[1-W] \\
w^{\prime} & =w\left[f_{w}(S) G(W)-\beta-k_{w}\right]+\alpha u[1-W] \delta^{-1} .
\end{aligned}
$$

The meaning of the terms in (3) are now described. Nutrient $S$ enters the chemostat in the feed stream at rate $D S^{0}$ and unused nutrient leaves at rate $D S$. Inside the chemostat, nutrient is consumed by both planktonic cells and wall attached cells. Planktonic cells $u$ grow in response to the uptake of nutrient, are washed out of the chemostat at rate $D u$ and suffer cell death rate $k$. Wall attached cells slough into the fluid at rate $\beta \delta w$ and a fraction $1-G(W)$ of the output $f_{w}(S) w$ of daughter cells of wall attached bacteria fail to find wall sites and thus enter the fluid. Finally, planktonic cells attach to the wall at rate $\alpha u[1-W]$. Wall attached cells grow (finding wall sites) at rate $f_{w}(S) G(W)$, suffer cell death $k_{w}$, are lost due to sloughing at rate $\beta$, and gain via the attachment of planktonic cells at rate $\alpha u[1-W]$.

The classical Monod (no wall attachment) model for planktonic cell growth (see e.g. Herbert et al [29]; Smith and Waltman [49]; Bailey and Ollis [2]) is recovered on setting $\alpha=w=0=k=k_{w}$.

If we set $w_{\max }=\infty$ making $W=0$, then we remove the hypothesis that the wall environment is finite and that daughter cells of wall adherent bacteria compete for wall sites. If also $G(0)=1$ then the model reduces to that of Pilyugin and Waltman [45].

$$
\begin{aligned}
S^{\prime} & =D\left(S^{0}-S\right)-\gamma^{-1}\left[u f_{u}(S)+\delta w f_{w}(S)\right] \\
u^{\prime} & =u\left[f_{u}(S)-D-k\right]+\beta \delta w-\alpha u \\
w^{\prime} & =w\left[f_{w}(S)-\beta-k_{w}\right]+\alpha u \delta^{-1} .
\end{aligned}
$$

A similar reduction can be made in the setting of a flow reactor in the sections to follow.

A mathematical analysis of the system (3) is given in Stemmons and Smith [52] in the case that $f_{u}=f_{w}, k=k_{w}=0$. The more general model considered here allows for different substrate uptake rates for planktonic and adherent cells and for nontrivial cell death rates.

The washout steady state

$$
(S, u, w)=\left(S^{0}, 0,0\right)
$$

always exists. Its stability, is determined by linearizing (3) about this equilibrium. The three-by-three Jacobian has one-one entry $-D$ below which are zeros. Therefore, stability depends on the eigenvalues of the $2 \times 2$ lower-right sub-matrix of the Jacobian given by

$$
A=\left(\begin{array}{rr}
f_{u}\left(S^{0}\right)-D-k-\alpha f_{w}\left(S^{0}\right)(1-G(0))+\beta \\
\alpha & f_{w}\left(S^{0}\right) G(0)-k_{w}-\beta
\end{array}\right) .
$$

This matrix is quasipositive (nonnegative off-diagonal entries), as are other matrices encountered throughout this work, and hence we will find it convenient to employ Perron-Frobenius theory [10]. The washout state is locally 
asymptotically stable if its eigenvalues have negative real part (they are real) and unstable if at least one is positive. We use the notation $s(A)$ for the largest eigenvalue of $A$. With this notation, the washout steady state is stable when $s(A)<0$ and unstable when $s(A)>0$. Although an exact (and ugly) expression may be written for $s(A)$ in terms of the entries, the following inequalities, the result of simple estimates, shed more light on the biology:

$$
\begin{aligned}
& \max \left\{f_{u}\left(S^{0}\right)-D-k-\alpha, f_{w}\left(S^{0}\right) G(0)-k_{w}-\beta\right\}< \\
& s(A) \leq \max \left\{f_{u}\left(S^{0}\right)-D-k, f_{w}\left(S^{0}\right)-k_{w}\right\} .
\end{aligned}
$$

Therefore, $s(A)<0$ and the washout state is (globally) stable if both

$$
f_{w}\left(S^{0}\right)-k_{w}<0 \text { and } f_{u}\left(S^{0}\right)-k-D<0 ;
$$

$s(A)>0$ and the washout state is unstable if either

$$
f_{w}\left(S^{0}\right) G(0)-k_{w}-\beta \geq 0
$$

or if

$$
f_{u}\left(S^{0}\right)-k-D-\alpha \geq 0 .
$$

Also, $s(A)>0$ if both

$$
f_{u}\left(S^{0}\right)-k-D>0 \text { and } f_{w}\left(S^{0}\right)-k_{w} \geq 0
$$

because if both (8) and (9) fail yet (10) holds, then the determinant of $A$ is negative.

The following result is proved in [35], relying on earlier work of [52].

Theorem 1. If $s(A)<0$, then the washout state is stable. It is globally attracting if, in addition to $s(A)<0$, either (7) or $f_{w}\left(S^{0}\right)-k_{w}>f_{u}\left(S^{0}\right)-k-D$ or $f_{u}=f_{w}$ holds.

If $s(A)>0$ then at least one nontrivial steady state $\left(S^{*}, u^{*}, w^{*}\right)$ exists and any nontrivial steady state satisfies

$$
u^{*}>0, w_{\max } \geq w^{*}>0,0<S^{*}<S^{0} .
$$

If $f_{u}(S)-k-D>0$ implies $f_{w}(S)-k_{w} \geq 0$ or if $f_{u}=f_{w}$, then at most one nontrivial steady state may exist. Finally, $s(A)>0$ implies that $u$ and $w$ persist: there exists $\epsilon>0$, independent of initial data, such that if $w(0)+$ $u(0)>0$, there is a $T>0$ (depending on the initial data) such that

$$
u(t)+w(t)>\epsilon, \quad t>T .
$$

In the case considered by Stemmons and Smith [52], sufficient conditions are given for the unique nontrivial steady state to attract all nontrivial initial data. The Pilyugin and Waltman system (4) is considerably simpler and global stability of the unique nontrivial equilibrium can be deduced in the special case that $f_{u}=f_{w}[45]$. Unfortunately, this condition is not realistic. 
Persistence of $u, w$ means that there is an initial-condition-independent lower bound for the ultimate bacterial density. In view of our inability to show that a nontrivial steady state is globally attracting, this says that at least the microbial population survives when $s(A)>0$. The proof of this assertion follows that given in Prop. 7.9 of [52].

Diekmann and Heesterbeek [20] (see Theorem 6.13) show that the stability modulus of $A$ can be related to the spectral radius of a related matrix and that this relation can be used to define a basic reproductive ratio which is more useful for interpreting our results. Specifically, they show that

$$
\operatorname{sign} s(A)=\operatorname{sign}\left[\rho\left(G T^{*}\right)-1\right]
$$

where $\rho$ denotes spectral radius and $T^{*}$ denotes the transpose of $T$. Here,

$$
G=\left(\begin{array}{cc}
f_{u}\left(S^{0}\right) & f_{w}\left(S^{0}\right)(1-G(0)) \\
0 & f_{w}\left(S^{0}\right) G(0)
\end{array}\right)
$$

and

$$
T=\frac{1}{\Delta}\left(\begin{array}{cc}
k_{w}+\beta & \alpha \\
\beta & D+k+\alpha
\end{array}\right)
$$

and

$$
\Delta=\left(\beta+k_{w}\right)(D+k+\alpha)-\alpha \beta=(D+k)\left(\beta+k_{w}\right)+k_{w} \alpha .
$$

The factors $G$ and $T$ have natural biological interpretations. If we identify indices $\{1,2\}=\{u, w\}$ then

$$
G_{i j}=\begin{aligned}
& \text { rate of production of daughter cells in compartment i by cells in } \\
& \text { compartment } \mathrm{j} \text { in washout environment }
\end{aligned}
$$

and

$$
T_{i j}=\begin{aligned}
& \text { mean time spent in state } \mathrm{j} \text { before washout or death by cell born in } \\
& \text { state i. }
\end{aligned}
$$

The matrix $T$ is calculated using the algorithm described in [30] (based on suggestions by S. Schrieber) identifying an appropriate continuous-time Markov chain model with rate matrix

$$
B=\left(\begin{array}{cc}
-D-k-\alpha & \beta \\
\alpha & -k_{w}-\beta
\end{array}\right)
$$

as described in Fig. 2. In [30] it is also argued that the mean residence time of a cell in the chemostat is given by

$$
\text { Mean Residence Time }=-\frac{1}{s(B)}=\rho(T) .
$$

Hence

$$
G T^{*}=\left(\begin{array}{cc}
f_{u}\left(S^{0}\right) T_{u u}+f_{w}\left(S^{0}\right)(1-G(0)) T_{u w} & f_{u}\left(S^{0}\right) T_{w u}+f_{w}\left(S^{0}\right)(1-G(0)) T_{w w} \\
f_{w}\left(S^{0}\right) G(0) T_{u w} & f_{w}\left(S^{0}\right) G(0) T_{w w}
\end{array}\right)
$$


gives the production of daughter cells into state $i$ by mother cell in state $j$ before removal by washout or cell death. Thus we are lead to define the Basic Reproductive Number $R_{0}$ as

$$
R_{0}=\rho\left(G T^{*}\right) .
$$

If $v=(\tau, 1-\tau)^{*}, 0<\tau<1$, is the normalized positive eigenvector corresponding to the simple eigenvalue $R_{0}$ for $G T^{*}$, then $G T^{*} v=R_{0} v$ and adding these two equations gives

$$
R_{0}=f_{u}\left(S^{0}\right)\left[\tau T_{u u}+(1-\tau) T_{w u}\right]+f_{w}\left(S^{0}\right)\left[\tau T_{u w}+(1-\tau) T_{w w}\right] .
$$

The first convex combination in brackets can be interpreted as a mean time spent by a cell (regardless of where born) in the fluid environment while the second is a mean time spent in the biofilm. Thus, $R_{0}$ can be interpreted as the replacement ratio in the washout state.

Our main result implies the existence of a threshold for survival of the organism in CSTR. Indeed, it is easy to see that $s(A)$ and $\rho\left(G T^{*}\right)$ are strictly increasing with supply substrate concentration $S^{0}$ and, obviously, $s(A)<0$ for very small $S^{0}$. Thus, there exists a critical substrate supply $0<S_{c}^{0} \leq \infty$ such that washout is stable if $S^{0}<S_{c}^{0}$ and unstable if $S^{0}>S_{c}^{0}$. However, the threshold may be so low as to be unobservable as it is likely that under most operating conditions, $k_{w}, \beta<<f_{w}\left(S^{0}\right)$ and therefore (8) holds. This explains the ubiquity of biofilms; whereas in a fluid environment organisms must grow fast enough to overcome washout, in biofilm they simply have to grow fast enough to exceed sloughing.

The lack of effectiveness of antimicrobial agents in controlling bacteria in biofilms has been noted in the literature. See for example Costerton et al. [17] and Stewart et al. [53, 54]. As noted in [17], resistance to antimicrobials is likely to have multiple causes, one being "the failure of an agent to penetrate the full depth of the biofilm. Polymeric substances like those that make up the matrix of a biofilm are known to retard the diffusion of antibiotics...". An extensive discussion may be found in [53]. In [35] we examined the extreme case of a biofilm layer which is impenetrable to an antibiotic $A$ introduced into the CSTR at concentration $A^{0}$. Following Stewart et al. [53], (see Equation (11.19)), we assume that the antibiotic increases planktonic death rates as its concentration increases: the death rate of planktonic bacteria $k=k(A)$ is an increasing function of $A$. Adherent bacteria are assumed to be unaffected because the antibiotic cannot penetrate the biofilm layer. Assuming that $A$ is not significantly depleted by its action, it satisfies

$$
A^{\prime}=D\left(A^{0}-A\right)
$$

and, obviously, 


$$
A(t) \rightarrow A^{0}, \quad t \rightarrow \infty
$$

Therefore the long-term effect of the antibiotic is essentially to adjust the death rate of planktonic bacteria to $k=k\left(A^{0}\right)$. However, it is easy to see from (6) that $s(A)$ is relatively insensitive to parameter $k$. If (8) holds, then $s(A)>0$ and the washout steady state is unstable regardless of the value of $k$. Therefore, we expect the bacterial population to survive in the CSTR regardless of the planktonic cell death rate. The density of planktonic bacteria may be driven quite low by the antibiotic but one expects that the wallattached cell density is largely unaffected. Thus we conclude that if (8) holds then introduction of antibiotic into the flow reactor will not be effective in eradicating the bacteria.

The effect of various biocides on biofilms is a topic of great interest and has spawned a great deal of mathematical modeling. See for example Hunt et al. [54] and the references therein and Cogan [15].

The left side of Figure 3, taken from [35], shows the formation of biofilm in CSTR. Both cell death rates are zero: $k=k_{w}=0$. Dependent and independent variables are scaled as follows: $\bar{t}=D t, \bar{S}=S / a, \bar{u}=u /(\gamma a), \bar{w}=$ $W=w / w_{\max }$. Initial data are chosen to simulate an inoculum of planktonic cells introduced into a sterile CSTR with substrate at equilibrium with inflow. They are: $\bar{S}=S^{0} / a, \bar{u}=10^{-5}, W=0$.

The time series can be decomposed into three stages. During the first, substrate remains near its initial value while an adherent population slowly accumulates. The second stage is characterized by a linear growth of the adherent population which rapidly depletes the substrate. Finally, a mature adherent population, fully occupying the wall $(w \approx 1)$, casts off significant numbers of planktonic cells. Note that one scaled time-unit equals $4 \mathrm{hr}$.

The right side of Figure 3, with the same initial data, shows the effect of a (very large) planktonic cell death rate of $25 \%$ of the dilution rate $(k=$ $\left..25 D, k_{w}=0\right)$ resulting from the introduction of antibiotic that does not penetrate the biofilm. The only noticeable effect is a smaller planktonic cell density; the antibiotic cannot inhibit biofilm formation.

\section{One-Dimensional Thin Tube Flow Reactor}

The flow model treated in this section was first formulated by Ballyk and Smith in [5] and later analyzed in more detail in [6]. It builds on earlier work in which wall growth was not treated in Ballyk, Jones, Le and Smith [3].

Consider a thin tube with inner circumference $C$ and cross-sectional area $A$ extending along the $x$-axis. See Figure 4 . The reactor occupies the portion of the tube from $x=0$ to $x=L$. It is fed with growth medium at a constant rate at $x=0$ by a laminar flow of fluid in the tube in the direction of increasing $x$ and at velocity $v$ (a constant). The external feed contains all nutrients in near optimal amounts except one, denoted by $S$, which is supplied in a 
constant, growth limiting concentration $S^{0}$. The flow carries medium, depleted nutrients, cells, and their byproducts out of the reactor at $x=L$. Nutrient $S$ is assumed to diffuse with diffusivity $d_{0}$ while free microbial cells are assumed to be capable of random run and tumble motion which can be modeled by diffusion with diffusivity (sometimes called random motility coefficient) $d$. See the classic monograph of Berg [9] for more on bacterial motility and our earlier work in [3]. Wall attached bacteria are assumed to be immobile. We assume negligible variation of free bacteria and nutrient concentration transverse to the axial direction of the tube.

The model accounts for the density of free bacteria (bacteria suspended in the fluid) $u(x, t)$, the density of wall-attached bacteria $w(x, t)$ and the density of nutrient $S(x, t)$. The total free bacteria at time $t$ is given by

$$
A \int_{0}^{L} u(x, t) d x
$$

and the total bacteria on the wall at time $t$ is given by

$$
C \int_{0}^{L} w(x, t) d x
$$

Let $\delta=C / A$, not to be confused with $\delta$ of the chemostat model. Then $S, u, w$ satisfy the following system of equations.

$$
\begin{aligned}
S_{t}= & d_{0} S_{x x}-v S_{x}-\gamma^{-1} u f_{u}(S)-\gamma^{-1} \delta w f_{w}(S) \\
u_{t}= & d u_{x x}-v u_{x}+u\left(f_{u}(S)-k\right)+\delta w f_{w}(S)(1-G(W)) \\
& -\alpha u(1-W)+\delta \beta w \\
w_{t}= & w\left(f_{w}(S) G(W)-k_{w}-\beta\right)+\alpha \delta^{-1} u(1-W),
\end{aligned}
$$

with boundary conditions

$$
\begin{aligned}
v S^{0} & =-d_{0} S_{x}(0, t)+v S(0, t), \quad S_{x}(L, t)=0 \\
0 & =-d u_{x}(0, t)+v u(0, t), \quad u_{x}(L, t)=0,
\end{aligned}
$$

and initial conditions

$$
S(x, 0)=S_{0}(x), \quad u(x, 0)=u_{0}(x), \quad w(x, 0)=w_{0}(x), \quad 0 \leq x \leq L .
$$

The boundary conditions (18), referred to as Danckwerts' boundary conditions by Aris [1], are often misunderstood. To understand their implications, it is useful to integrate the equations over the domain $[0, L]$ to obtain the mass balance 


$$
\begin{aligned}
\frac{d}{d t} A \int_{0}^{L} S d x= & A \int_{0}^{L} d_{0} S_{x x}-v S_{x}-\gamma^{-1}\left[u f_{u}(S)+\delta w f_{w}(S)\right] d x \\
= & -A\left[-d_{0} S_{x}(L, t)+v S(L, t)\right]+A\left[-d_{0} S_{x}(0, t)+v S(0, t)\right] \\
& -A \int_{0}^{L} \gamma^{-1}\left[u f_{u}(S)+\delta w f_{w}(S)\right] d x \\
= & -v A S(L, t)+v A S^{0}-\int_{0}^{L} \gamma^{-1}\left[A u f_{u}(S)+C w f_{w}(S)\right] d x .
\end{aligned}
$$

Thus, the change in the total amount of nutrient in the tube is due to the flow bringing fresh nutrient in at $x=0$ at rate $v A S^{0}$, taking out unused nutrient at $x=L$ at rate $v A S(L, t)$ and due to the consumption of nutrient by planktonic and wall attached cells in the reactor. By contrast, a similar calculation for the planktonic bacteria $u$ yields no counterpart to the influx of fresh nutrient into the reactor at $x=0$ since we assume the inflow is sterile. In summary, the Danckwerts' boundary conditions say that advection alone mediates the interaction of the reactor with the external environment. See $[2,39,56]$ for other uses of these boundary conditions and particularly the latter for alternative conditions.

System (17)-(19) has a trivial steady state

$$
S \equiv S^{0}, \quad u=w \equiv 0
$$

which we refer to as the 'washout steady state' since no organisms are present. The linearization of (17)-(19) about the washout steady state is given by:

$$
\begin{aligned}
S_{t}= & d_{0} S_{x x}-v S_{x}-\gamma^{-1} u f_{u}\left(S^{0}\right)-\delta \gamma^{-1} w f_{w}\left(S^{0}\right) \\
u_{t}= & d u_{x x}-v u_{x}+u\left(f_{u}\left(S^{0}\right)-k\right)+\delta w f_{w}\left(S^{0}\right)(1-G(0)) \\
& -\alpha u+\delta \beta w \\
w_{t}= & w\left(f_{w}\left(S^{0}\right) G(0)-k_{w}-\beta\right)+\delta^{-1} \alpha u
\end{aligned}
$$

with the homogeneous boundary conditions:

$$
\begin{aligned}
& 0=-d_{0} S_{x}(0, t)+v S(0, t), \quad S_{x}(L, t)=0 \\
& 0=-d u_{x}(0, t)+v u(0, t), \quad u_{x}(L, t)=0 .
\end{aligned}
$$

Introducing $(S, u, w)=\exp (\lambda t)(\bar{S}(x), \bar{u}(x), \bar{w}(x))$ into $(20)$, we arrive at the eigenvalue problem relevant for the stability of the washout steady state

$$
\begin{aligned}
\lambda \bar{S}= & d_{0} \bar{S}^{\prime \prime}-v \bar{S}^{\prime}-\gamma^{-1} \bar{u} f_{u}\left(S^{0}\right)-\delta \gamma^{-1} \bar{w} f_{w}\left(S^{0}\right) \\
\lambda \bar{u}= & d \bar{u}^{\prime \prime}-v \bar{u}^{\prime}+\bar{u}\left(f_{u}\left(S^{0}\right)-k\right)+\delta \bar{w} f_{w}\left(S^{0}\right)(1-G(0)) \\
& -\alpha \bar{u}+\delta \beta \bar{w} \\
\lambda \bar{w}= & \bar{w}\left(f_{w}\left(S^{0}\right) G(0)-k_{w}-\beta\right)+\delta^{-1} \alpha \bar{u}
\end{aligned}
$$


with

$$
\begin{aligned}
& 0=-d_{0} \bar{S}^{\prime}(0)+v \bar{S}(0), \quad \bar{S}^{\prime}(L)=0 \\
& 0=-d \bar{u}^{\prime}(0)+v \bar{u}(0), \quad \bar{u}^{\prime}(L)=0 .
\end{aligned}
$$

It turns out that the eigenvalues of (21)-(22) determine the stability of the washout steady state despite the fact that the spectrum of the differentialalgebraic operator appearing on the righthand side of (21), with the boundary conditions determining its domain, may not consist solely of eigenvalues.

The following result is proved in [6].

Theorem 2. Let

$$
\hat{A}=\left(\begin{array}{rr}
f_{u}\left(S^{0}\right)-k-\alpha-\frac{v}{L} \lambda_{\bar{d}} f_{w}\left(S^{0}\right)(1-G(0))+\beta \\
\alpha & f_{w}\left(S^{0}\right) G(0)-k_{w}-\beta
\end{array}\right) .
$$

where $\bar{d}=d / L v$ and $-\lambda_{\bar{d}}<0$ is the largest eigenvalue of the scaled eigenvalue problem

$$
\begin{aligned}
\lambda \phi & =\bar{d} \phi^{\prime \prime}-\phi^{\prime} \\
0 & =-\bar{d} \phi^{\prime}(0)+\phi(0), \quad \phi^{\prime}(1)=0 .
\end{aligned}
$$

Let $s(\hat{A})$ be the stability modulus, i.e., the largest of the distinct real eigenvalues of matrix $\hat{A}$. If $s(\hat{A})<0$ then all eigenvalues of (21) are negative and the washout steady state is asymptotically stable; the washout steady state is unstable whenever $s(\hat{A})>0$.

Note the similarity of the two stability determining matrices (5) and (23). The only difference is that $D$ in (5) is replaced by $\frac{v}{L} \lambda_{\bar{d}}$ in (23). The term $\frac{v}{L} \lambda_{\bar{d}}$ should be viewed as an effective washout or removal rate from the bio-reactor. For the CSTR, $1 / D$ is the mean residence time in the chemostat so there is a close correspondence between the two matrices.

It may seem striking that stability boils down to the sign of the leading eigenvalue of a $2 \times 2$ matrix exactly as in the case of the continuous culture model. We would argue that it is quite natural on biological grounds. There are two habitats for the bacteria, the wall and the bulk fluid. To survive, the organism must be able to establish itself in at least one of the habitats sufficiently well to overcome the constant leakage to the other, possibly less suitable, habitat.

It is evident that the basic reproductive number defined in (16) can be adopted to the tubular reactor. All formulas obtained for CSTR carry over to the tubular reactor by merely replacing the dilution rate $D$ by its counterpart $\frac{v}{L} \lambda_{\bar{d}}$.

The following global results are special cases of Theorem 3.2 and Theorem 4.1 in $[6]$. 
Theorem 3. If $s(\hat{A})<0$ and $f_{w}\left(S^{0}\right)-k_{w}>f_{u}\left(S^{0}\right)-k-\lambda_{d}$ then

$$
\int_{0}^{1}[u(x, t)+w(x, t)] d x \rightarrow 0, \quad t \rightarrow \infty .
$$

If $s(\hat{A})>0$ and

$$
f_{w}\left(S^{0}\right) G(0)-k_{w}-\beta \neq 0,
$$

then there exists a steady state solution $(S, u, w)$ satisfying

$$
\begin{aligned}
& 0<S(x)<S^{0}, \quad S^{\prime}(x)<0, \quad u(x)>0, \text { and } \\
& 0<w(x)<w_{\max }, \quad 0 \leq x \leq L .
\end{aligned}
$$

Figure 5 from [6] depicts the steady state solution of (17)-(19) where cell death rates are zero. Observe that wall attached cells fully occupy the front lip near $x=0$ of the tube $\left(w_{\max }=1\right)$, dropping rapidly to zero when nutrient is depleted downstream in the $150 \mathrm{~cm}$ tube. The shape of the suspended (planktonic) cell density profile implies that these cells are being sloughed off the wall at a rate just balancing their washout.

\subsection{Advection Dominated Flow Reactor}

If diffusion and cell motility are small compared to advection in (17)-(19) then we obtain the hyperbolic system studied by Jones and Smith in [36].

$$
\begin{aligned}
S_{t}+v S_{x}= & -\gamma^{-1} u f_{u}(S)-\gamma^{-1} \delta w f_{w}(S) \\
u_{t}+v u_{x}= & u\left(f_{u}(S)-k\right)+\delta w f_{w}(S)(1-G(W)) \\
& -\alpha u(1-W)+\delta \beta w \\
w_{t}= & w\left(f_{w}(S) G(W)-k_{w}-\beta\right)+\alpha \delta^{-1} u(1-W)
\end{aligned}
$$

Boundary conditions, representing the influx of nutrient in a sterile feed are given by

$$
\begin{aligned}
v S^{0} & =v S(0, t), \\
0 & =v u(0, t),
\end{aligned}
$$

and initial conditions are

$$
S(x, 0)=S_{0}(x), \quad u(x, 0)=u_{0}(x), \quad w(x, 0)=w_{0}(x), \quad 0 \leq x \leq L .
$$

We assume that $S_{0}, u_{0}, w_{0}$ are nonnegative, continuous and $w_{0} \leq w_{\max }$.

It is shown in [36] that the system (25)-(27) has a unique global continuous solution provided that $S_{0}, u_{0} \geq 0$ and $0 \leq w_{0} \leq w_{\max }$ and the compatibility conditions $S_{0}(0)=S^{0}$ and $u_{0}(0)=0$ hold. A more thorough treatment of 
well-posedness for a general class of hyperbolic systems including (25)-(27) is carried out in [48].

As above, the focus is on the stability of the washout equilibrium solution

$$
S=S^{0}, \quad u=w=0 .
$$

Remarkably, the wall-attached population density $W(t) \equiv w(0, t) / w_{\max }$ at $x=0$ where nutrient concentration is highest can be computed from the scalar ordinary differential equation (since $u(0, t)=0$ ):

$$
\frac{d W}{d t}=W\left[f_{w}\left(S^{0}\right) G(W)-k_{w}-\beta\right] .
$$

As $G$ is strictly decreasing on $[0,1]$, it is easily seen that

$$
f_{w}\left(S^{0}\right) G(0)-k_{w}-\beta<0 \Longrightarrow W(t) \rightarrow 0
$$

and if $W(0)>0$ then

$$
f_{w}\left(S^{0}\right) G(0)-k_{w}-\beta>0 \Longrightarrow W(t) \rightarrow W^{*}
$$

where $W^{*}$ is the unique solution of

$$
f_{w}\left(S^{0}\right) G(W)-k_{w}-\beta=0 .
$$

This motivates the following result, proved in [36], which establishes that the sign of $f_{w}\left(S^{0}\right) G(0)-k_{w}-\beta$ determines the asymptotic behavior of (25)-(27).

Theorem 4. If

$$
f_{w}\left(S^{0}\right) G(0)-k_{w}-\beta<0
$$

holds then every solution of (25)-(27) converges to the washout steady state, uniformly in $x \in[0, L]$.

If

$$
f_{w}\left(S^{0}\right) G(0)-k_{w}-\beta>0
$$

then there is a unique steady state solution $(\bar{S}, \bar{u}, \bar{w})$ satisfying

$$
0<\bar{S}(x)<S^{0}, 0<\bar{u}(x), 0<\bar{w}(x) \leq w_{\max }, 0<x \leq L,
$$

where $W^{*}=\bar{w}(0) / w_{\max }$. Moreover, it is asymptotically stable in the linear approximation.

Again we see that biofilm bacteria simply must grow fast enough to out pace any losses due to cell death and sloughing in order to survive. The steady state plots in Figure 6 show the strong decline in nutrient concentration as one moves downstream and the wall adherent cells occupying the front lip near $x=0$ of the tubular reactor. Planktonic cells are sloughed off this front lip at a rate which balances washout. Parameter values for the simulation in Figure 6 are as follows: $k_{w}=.05, \alpha=\beta=.2, \delta=10.0, k=.2, f(S)=$ $f_{w}(S)=S /(1+S)$. Initial data are $S_{0}(x)=1, u_{0}(x)=x, w_{0}(x)=0$. 


\subsection{Mobile Wall-Adherent Cells}

Smith and Zhao [50] consider the case that wall-adherent bacteria are also mobile. The equations now become more mathematically tractable since the added diffusion term has a smoothing effect. The system is given by:

$$
\begin{aligned}
S_{t}= & d_{0} S_{x x}-v S_{x}-\gamma^{-1} u f_{u}(S)-\gamma^{-1} \delta w f_{w}(S) \\
u_{t}= & d u_{x x}-v u_{x}+u(f(S)-k)+\delta w f_{w}(S)(1-G(W)) \\
& -\alpha u(1-W)+\delta \beta w \\
w_{t}= & d_{1} w_{x x}+w\left(f_{w}(S) G(W)-k_{w}-\beta\right)+\alpha \delta^{-1} u(1-W),
\end{aligned}
$$

where $d_{1}$ denotes the motility of wall-attached bacteria, with boundary conditions

$$
\begin{aligned}
v S^{0} & =-d_{0} S_{x}(0, t)+v S(0, t), \quad S_{x}(L, t)=0 \\
0 & =-d u_{x}(0, t)+v u(0, t), \quad u_{x}(L, t)=0 \\
0 & =w_{x}(0, t)=w_{x}(L, t)
\end{aligned}
$$

and initial conditions

$$
S(x, 0)=S_{0}(x), \quad u(x, 0)=u_{0}(x), \quad w(x, 0)=w_{0}(x), \quad 0 \leq x \leq L .
$$

This system is well-posed for continuous nonnegative initial data and induces a semiflow with a compact global attractor on the nonnegative cone of the space of triples of continuous functions on $[0, L]$. See Theorem 3.2 in [50].

The focus is again on the stability of the washout equilibrium

$$
S=S^{0}, \quad u=w=0 .
$$

The associated eigenvalue problem is given by

$$
\begin{aligned}
\lambda \bar{S}= & d_{0} \bar{S}_{x x}-v \bar{S}_{x}-\gamma^{-1} \bar{u} f_{u}\left(S^{0}\right)-\delta \gamma^{-1} \bar{w} f_{w}\left(S^{0}\right) \\
\lambda \bar{u}= & d \bar{u}_{x x}-v \bar{u}_{x}+\bar{u}\left(f\left(S^{0}\right)-k\right)+\delta \bar{w} f_{w}\left(S^{0}\right)(1-G(0)) \\
& -\alpha \bar{u}+\delta \beta \bar{w} \\
\lambda \bar{w}= & d_{1} w_{x x}+\bar{w}\left(f_{w}\left(S^{0}\right) G(0)-k_{w}-\beta\right)+\delta^{-1} \alpha \bar{u}
\end{aligned}
$$

with

$$
\begin{aligned}
& 0=-d_{0} \bar{S}_{x}(0)+v \bar{S}(0), \quad \bar{S}_{x}(L)=0 \\
& 0=-d \bar{u}_{x}(0)+v \bar{u}(0), \quad \bar{u}_{x}(L)=0 \\
& 0=\bar{w}_{x}(0)=\bar{w}_{x}(L) .
\end{aligned}
$$

The stability of washout is determined by a dominant eigenvalue but note that this eigenvalue problem decouples in the sense that the last two equations are independent of the first and therefore, we need to consider dominant eigenvalues both for this subsystem as well as for the full eigenvalue problem. The following result is proved in [50]. 
Lemma 1. There exists a dominant real eigenvalue $\Lambda$ of (32)-(33). That is, $\Re \lambda<\Lambda$ for all other eigenvalues $\lambda$ of (32). The washout steady state is asymptotically stable if $\Lambda<0$ and unstable if $\Lambda>0 . \Lambda$ is related to the dominant eigenvalue $\Gamma$ of the last two equations of (32)-(33) as follows. If $\Gamma>-\lambda_{d_{0}}$, which holds if $f_{w}\left(S^{0}\right) G(0)-k_{w}-\beta>-\lambda_{d_{0}}$, then

$$
f_{w}\left(S^{0}\right) G(0)-k_{w}-\beta<\Lambda=\Gamma<s(\tilde{A})
$$

where

$$
\tilde{A}=\left(\begin{array}{cr}
f_{u}\left(S^{0}\right)-k-\alpha & f_{w}\left(S^{0}\right)(1-G(0))+\beta \\
\alpha & f_{w}\left(S^{0}\right) G(0)-k_{w}-\beta
\end{array}\right)
$$

and $\lambda_{d_{0}}$ is defined in Theorem 2. If $\Gamma \leq-\lambda_{d_{0}}$, then $\Lambda=-\lambda_{d_{0}}$.

As a special case, note that

$$
f_{w}\left(S^{0}\right) G(0)>k_{w}+\beta
$$

implies $\Lambda>0$ and hence the instability of the washout state.

As in the case when wall adherent bacteria are assumed to be immobile, one can give conditions for the global stability of the washout equilibrium (see Proposition 4.4 in [50]) but they are not sharp. We conjecture that $\Lambda<0$ is sufficient for this.

The main result of [50] follows.

Theorem 5. Assume $\Lambda>0$. Then system (29)-(31) has at least one positive steady state:

$$
0<S(x)<S^{0}, 0<u(x), 0<w(x) \leq w_{\max }
$$

Furthermore, it is uniformly persistent in the sense that there exists $\eta>0$ such that for any nonnegative continuous initial data $\left(S_{0}, u_{0}, w_{0}\right)$, with at least one of $u_{0}(\cdot)$ and $w_{0}(\cdot)$ not identically zero, there exists $T_{0}=T_{0}\left(S_{0}, u_{0}, w_{0}\right)>0$ such that the solution $(S, u, w)$ satisfies

$$
S(x, t) \geq \eta, \quad u(x, t) \geq \eta, \quad w(x, t) \geq \eta, \quad x \in[0, L], \quad t \geq T_{0} .
$$

\section{Three-Dimensional Flow Reactor}

The three dimensional model is substantially more complicated because the fluxes between adherent and planktonic compartments of the model appear as part of the (nonlinear) boundary conditions. Our treatment here follows Jones et al. [33]. Consider a cylindrical tube

$$
\Omega=\left\{(x, y, z) \in \mathbb{R}^{3}: 0<\mathrm{x}<\mathrm{L}, \quad 0 \leq \mathrm{r}^{2}=\mathrm{y}^{2}+\mathrm{z}^{2}<\mathrm{R}^{2}\right\}
$$

under steady flow with velocity profile 


$$
v(r)=V_{\max }\left[1-(r / R)^{2}\right] .
$$

The equations for nutrient density $S=S(x, y, z, t)$, planktonic biomass density $u=u(x, y, z, t)$ and the areal density of wall-attached cells $w=$ $w(x, y, z, t)$ on the radial boundary $(r=R)$ for a single strain are given by:

$$
\begin{aligned}
& S_{t}=d_{x}^{S} S_{x x}+d_{r}^{S}\left[S_{y y}+S_{z z}\right]-v(r) S_{x}-\gamma^{-1} u f_{u}(S) \\
& u_{t}=d_{x}^{u} u_{x x}+d_{r}^{u}\left[u_{y y}+u_{z z}\right]-v(r) u_{x}+u\left(f_{u}(S)-k\right)
\end{aligned}
$$

for $(x, y, z) \in \Omega$. Substrate diffusivity in the axial direction is denoted by $d_{x}^{S}$ and in the radial direction by $d_{r}^{S}$. Planktonic bacteria are assumed to follow a random run and tumble motion which can be modeled by diffusion (Berg [9]). Motility coefficients $d_{x}^{u}$ and $d_{r}^{u}$ in the axial and radial directions are prescribed.

Wall-adherent cells are assumed to be immobile. Growth of bacteria on the wall $r=R$ is described by

$$
w_{t}=w\left[f_{w}(S) G(W)-k_{w}-\beta\right]+\alpha u(1-W) .
$$

(The units of $\alpha$ are now $l t^{-1}$ )

Danckwerts' boundary conditions describe the interface conditions between up-stream and down-stream flow and the reactor. They are as follows : at $x=0$ :

$$
\begin{array}{r}
v(r) S^{0}=-d_{x}^{S} S_{x}+v(r) S \\
0=-d_{x}^{u} u_{x}+v(r) u,
\end{array}
$$

at $x=L$ :

$$
S_{x}=u_{x}=0 .
$$

These conditions reflect the assumption that upstream flow brings sterile nutrient at concentration $S^{0}$ into the reactor at $x=0$ and flushes out planktonic cells and unused nutrient at $x=L$. The radial boundary conditions at $r=R$ reflect important biological considerations:

$$
\begin{aligned}
& -d_{r}^{S} S_{r}=\gamma^{-1} w f_{w}(S) \\
& -d_{r}^{u} u_{r}=\alpha u(1-W)-w\left[f_{w}(S)(1-G(W))+\beta\right] .
\end{aligned}
$$

They describe the fluxes of nutrient and biomass between the fluid and wall environment. The first describes the flux of nutrient from the fluid to the wall environment due to consumption by wall-attached bacteria. The first term in the second equation represents the flux of biomass from the fluid to the wall due to passive attraction of planktonic cells to the wall; the second term represents flux in the opposite direction caused by a fraction of the progeny of wall-attached cells being forced into the fluid.

In addition, $S, u, w$ satisfy (non-negative) initial conditions at $t=0$ :

$$
\begin{gathered}
S(x, y, z, 0)=S_{0}(x, y, z) \\
u(x, y, z, 0)=u_{0}(x, y, z) \\
w(x, y, z, 0)=w_{0}(x, y, z),
\end{gathered}
$$


where $S_{0}, u_{0}, w_{0}$ are continuous. Existence of a unique weak solution of the system (35)-(40) and its Hölder continuity is established in [33].

It will also be of interest to allow the initial "charging" of the reactor with microbes to take place via the boundary condition at $x=0$ by replacing zero on the left side of (37) by $v(r) u^{0}(t)$, where $u^{0}(t) \equiv u^{0}$, a constant, on $0 \leq t \leq t_{0}$ and $u^{0}(t)=0, t \geq t_{0}$.

In order to clarify that the system (35)-(40) captures the intended mass transfer, we integrate the $S$ equation over $\Omega$ and use cylindrical coordinates $(r, \theta, x)$, to obtain

$$
\begin{aligned}
& \frac{\partial}{\partial t} \iiint_{\Omega} S d V= \\
= & \iiint_{\Omega}\left(\left[d_{x}^{S} S_{x x}-v(r) S_{x}\right]+d_{r}^{S}\left[\frac{1}{r}\left(r S_{r}\right)_{r}+\frac{1}{r^{2}} S_{\theta \theta}\right]\right) r d r d x d \theta \\
& -\iiint_{\Omega} \gamma^{-1} u f_{u}(S) d V \\
= & \left.\int_{0}^{2 \pi} \int_{0}^{R}\left(d_{x}^{S} S_{x}-v(r) S\right)\right|_{x=L} r d r d \theta-\left.\int_{0}^{2 \pi} \int_{0}^{R}\left(d_{x}^{S} S_{x}-v(r) S\right)\right|_{x=0} r d r d \theta \\
& +\left.R d_{r}^{S} \int_{0}^{2 \pi} \int_{0}^{L} S_{r}\right|_{r=R} d x d \theta-\iiint_{\Omega} \gamma^{-1} u f_{u}(S) d V \\
= & -\left.\int_{0}^{2 \pi} \int_{0}^{R} v(r) S\right|_{x=L} d r d \theta+\int_{0}^{2 \pi} \int_{0}^{R} v(r) S^{0} d r \\
& -\left.R \int_{0}^{2 \pi} \int_{0}^{L} \gamma^{-1} w f_{w}(S)\right|_{r=R} d x d \theta-\iiint_{\Omega} \gamma^{-1} u f_{u}(S) d V
\end{aligned}
$$

The rate of change of substrate in $\Omega$ is the flux of substrate into $\Omega$ at $x=0$ minus the flux of substrate out of $\Omega$ at $x=L$ minus substrate consumed by wall-adherent organisms on the inside wall of the cylinder $r=R$ and minus substrate consumed by planktonic organisms in $\Omega$.

Similarly, integrating the $u$ equation leads to 


$$
\begin{aligned}
& \frac{\partial}{\partial t} \iiint_{\Omega} u d V= \\
= & \iiint_{\Omega}\left(\left[d_{x}^{u} u_{x x}-v(r) u_{x}\right]+d_{r}^{u}\left[\frac{1}{r}\left(r u_{r}\right)_{r}+\frac{1}{r^{2}} u_{\theta \theta}\right]\right) r d r d x d \theta \\
& +\iiint_{\Omega} u\left[f_{u}(S)-k\right] d V \\
= & \left.\int_{0}^{2 \pi} \int_{0}^{R}\left(d_{x}^{u} u_{x}-v(r) u\right)\right|_{x=L} r d r d \theta-\left.\int_{0}^{2 \pi} \int_{0}^{R}\left(d_{x}^{u} u_{x}-v(r) u\right)\right|_{x=0} r d r d \theta \\
& +\left.R d_{r}^{u} \int_{0}^{2 \pi} \int_{0}^{L} u_{r}\right|_{r=R} d x d \theta+\iiint_{\Omega} u\left[f_{u}(S)-k\right] d V \\
= & \left.\int_{0}^{2 \pi} \int_{0}^{R} v(r) u\right|_{x=L} r d r d \theta \\
& +\left.R \int_{0}^{2 \pi} \int_{0}^{L}\left(w\left[f_{w}(S)(1-G(W))+\beta\right]-\alpha u(1-W)\right)\right|_{r=R} d x d \theta \\
& +\iint_{\Omega} u\left[f_{u}(S)-k\right] d V
\end{aligned}
$$

Planktonic biomass increases due to net growth, due to the sloughing of walladherent cells into the fluid, and due to the failure of a fraction of the daughter cells of wall attached bacteria to find wall sites and it decreases due to attachment of planktonic cells to the lateral surface and to washing out at $x=L$.

For brevity, we let $L^{S}$ and $L^{u}$ denote the differential operators for the $S$ and $u$ equations so they become:

$$
\begin{aligned}
& S_{t}=L^{S} S-\gamma^{-1} u f_{u}(S) \\
& u_{t}=L^{u} u+u\left(f_{u}(S)-k\right) .
\end{aligned}
$$

Our aim now is to perform a linear stability analysis of the washout equilibrium:

$$
S=S^{0}, u=w=0
$$

and to show the existence of a nontrivial steady state in which $u, w>0$ under suitable conditions. The linear variational equation about the washout equilibrium is

$$
\begin{aligned}
S_{t} & =L^{S} S-\gamma^{-1} u f_{u}\left(S^{0}\right) \\
u_{t} & =L^{u} u+u\left[f_{u}\left(S^{0}\right)-k\right] \\
w_{t} & =w\left[f_{w}\left(S^{0}\right) G(0)-k_{w}-\beta\right]+\alpha u
\end{aligned}
$$

together with homogeneous boundary conditions $x=0$ (formally, set $S^{0}=0$ in (37)) and $x=L$ and radial boundary conditions on $r=R$ :

$$
\begin{aligned}
& 0=d_{r}^{S} S_{r}+\gamma^{-1} w f_{w}\left(S^{0}\right) \\
& 0=d_{r}^{u} u_{r}+\alpha u-w\left[f_{w}\left(S^{0}\right)(1-G(0))+\beta\right] .
\end{aligned}
$$


The associated eigenvalue problem, obtained by seeking solutions $\hat{S}=$ $e^{\lambda t} S(x, y, z)$ (and similarly for other variables), is

$$
\begin{aligned}
\lambda S & =L^{S} S-\gamma^{-1} u f_{u}\left(S^{0}\right) \\
\lambda u & =L^{u} u+u\left[f_{u}\left(S^{0}\right)-k\right] \\
\lambda w & =w\left[f_{w}\left(S^{0}\right) G(0)-k_{w}-\beta\right]+\alpha u
\end{aligned}
$$

together with the above boundary conditions.

We have the following from [33].

Proposition 1. The eigenvalue $\lambda^{*}$ of (43) with the largest real part is real, simple and satisfies $\lambda^{*}>f_{w}\left(S^{0}\right) G(0)-k_{w}-\beta$. It belongs to the interval with endpoints $f_{w}\left(S^{0}\right)-k_{w}$ and $f_{u}\left(S^{0}\right)-k-\frac{L}{V_{\max }} \lambda$, where $-\lambda<0$ is the principal eigenvalue of the (scaled $\bar{x}=x / L, \bar{r}=r / R$ ) eigenvalue problem:

$$
\begin{aligned}
\lambda u & =\theta_{x} u_{\bar{x} \bar{x}}-\left(1-\bar{r}^{2}\right) u_{\bar{x}}+\theta_{r} \bar{r}^{-1}\left(\bar{r} u_{\bar{r}}\right)_{\bar{r}}, \\
0 & =-\theta_{x} u_{\bar{x}}+\left(1-\bar{r}^{2}\right) u, \quad \bar{x}=0 \\
0 & =u_{\bar{x}}, \quad \bar{x}=1 \\
u_{\bar{r}} & =0, \quad \bar{r}=1
\end{aligned}
$$

where $\theta_{x}=\left(d_{x}^{u} / L^{2}\right)\left(L / V_{\max }\right), \theta_{r}=\left(d_{r}^{u} / R^{2}\right)\left(L / V_{\max }\right)$. The washout state is stable in the linear approximation if $\lambda^{*}<0$ and unstable if $\lambda^{*}>0$.

As a result of the inequalities above, we see that $\lambda^{*}<0$ and the washout state is stable if

$$
f_{w}\left(S^{0}\right)-k_{w}<0 \text { and } f_{u}\left(S^{0}\right)-k-\frac{L}{V_{\max }} \lambda<0 ;
$$

$\lambda^{*}>0$ and the washout state is unstable if either

$$
f_{w}\left(S^{0}\right) G(0)-k_{w}-\beta \geq 0
$$

or if

$$
f_{u}\left(S^{0}\right)-k-\frac{L}{V_{\max }} \lambda>0 \text { and } f_{w}\left(S^{0}\right)-k_{w}>0 .
$$

The reader will observe the similarity in the CSTR and PFR results. Obviously, $\frac{L}{V_{\max }} \lambda$ is an effective dilution rate for PFR.

We conjecture that the washout state is globally stable if $\lambda^{*}<0$ as in the case of the CSTR. It can be shown (Jones et al. [33]) that if (44) holds then the washout state is globally attracting in the sense that

$$
\lim _{t \rightarrow \infty}\left(\iiint_{\Omega} u d V+\int_{0}^{2 \pi} \int_{0}^{L} w R d \theta d x\right)=0 .
$$

The steady state equations are 


$$
\begin{aligned}
& 0=L^{S} S-\gamma^{-1} u f_{u}(S) \\
& 0=L^{u} u+u\left[f_{u}(S)-k\right], \quad \text { in } \Omega \\
& 0=w\left[f_{w}(S) G(W)-k_{w}-\beta\right]+\alpha u(1-W), \quad \text { on } r=R,
\end{aligned}
$$

with boundary conditions $(37),(38,(39)$. We summarize our main result for PFR below. It follows from Proposition 3.4 and Theorem 3.5 in [33] and Proposition $4.2(\mathrm{~b})$ in [32].

Theorem 6. If $\lambda^{*}<0$, then the washout state is stable in the linear approximation; it is globally attracting if (44) holds. If $\lambda^{*}>0$ and the non-degeneracy condition $b=f_{w}\left(S^{0}\right) G(0)-k_{w}-\beta \neq 0$ holds then there exists a radially symmetric steady state solution $(S, u, w)$ of (46) satisfying (in cylindrical coordinates)

$$
0<S(x, r) \leq S^{0}, \quad u(x, r)>0, \text { and } 0<w(x) \leq w_{\max } .
$$

A persistence result similar to that described above for CSTR is also proved in [33] if $\lambda^{*}>0$ and certain additional assumptions hold. 
The initial conditions for wall-attached bacteria and planktonic bacteria are $S^{0}=S_{0}, w_{0}=0$, and $u_{0}=0$, respectively. The reactor is charged with bacteria by replacing the original boundary condition on the reactor entrance with $v(r) u^{0}(t)=-d_{x}^{u} u_{x}+v(r) u$, where $u^{0}(t)=10^{-9} U_{5}(t)$ and $U_{5}(t)$ is the step function of unit height turning to zero at $T=5$. This mimics the introduction of an inoculum of planktonic cells into a bacteria-free PFR. Note the physical time scale is $L / V_{\max }=40 \mathrm{hrs}$. In order to provide a steady-state profile, the equations were integrated to time $T=1500$ hours at which point no further change could be detected, as can be seen in Fig. 4 (left).

The PFR time series displayed in Figure 7 (top) is roughly similar to that in Fig. 2 for CSTR but note the log-log scale used to accommodate the range of the data. An initial period of unchanged total substrate where wall adherent and planktonic cells accumulate is followed by a rapid decline in substrate and a slowing of the rate of increase of adherent and planktonic cells. As the substrate levels off, the wall adherent population does too while the planktonic population level declines somewhat as steady-state is achieved.

As noted in section 2.2, wall adherent bacterial cells may have flagella and consequently have limited motility on the cylindrical surface $r=R$. Furthermore, including random cell motility on this surface should facilitate the realization of the biofilm system as a well-behaved semiflow in a suitable function space possessing a compact global attractor just as it did in section 2.2. For that reason we are lead to introduce (35)-(40) where equation (36) is replaced by:

$$
\begin{aligned}
w_{t} & =d^{w}\left[R^{-2} w_{\theta \theta}+w_{x x}\right]+w\left[f_{w}(S) G(W)-k_{w}-\beta\right]+\alpha u(1-W) \\
w_{x}(x, \theta) & =0, x=0, L \\
w(x, \theta) & =w(x, \theta+2 \pi)
\end{aligned}
$$

In future work we will extend the analysis in section 2.2 to this system.

\section{Mixed Culture}

A mixed culture model treating competition for substrate and wall colonization sites in an advection-dominated flow reactor was considered by Jones and Smith in [36]. Here, we follow [32] where both advection and diffusion are included in a three dimensional flow reactor. A flow reactor amounts to a section of the cylindrical tube $\Omega$ in which a steady flow of fluid in the direction of increasing $x$ is imposed. The flow carries fresh nutrient at concentration $S^{0}$ into the reactor across the $x=0$ interface and carries unused nutrient and bacteria out of the reactor across the $x=L$ interface. The equations describing nutrient density $S=S(x, y, z, t)$ and biomass density $u^{i}=u^{i}(x, y, z, t)$ of bacteria of strain $i$ in the fluid (i.e., in $\Omega$ ) are given by: 


$$
\begin{aligned}
& S_{t}=d_{x}^{S} S_{x x}+d_{r}^{S}\left[S_{y y}+S_{z z}\right]-v(r) S_{x}-\sum_{i} \gamma_{i}^{-1} u^{i} f_{u i}(S) \\
& u_{t}^{i}=d_{x}^{i} u_{x x}^{i}+d_{r}^{i}\left[u_{y y}^{i}+u_{z z}^{i}\right]-v(r) u_{x}^{i}+u^{i}\left(f_{u i}(S)-k_{i}\right)
\end{aligned}
$$

for $(x, y, z) \in \Omega$. The areal density of wall-attached cells of strain $i$ on the radial boundary $r=R$ of $\Omega$, denoted by $w^{i}=w^{i}(x, y, z, t)$, satisfy the equations by

$$
w_{t}^{i}=w^{i}\left[f_{w i}(S) G_{i}(W)-k_{w i}-\beta_{i}\right]+\alpha_{i} u^{i}(1-W) .
$$

The specific growth rate of strain $i$ in the fluid $f_{u i}(S)$ and on the wall $f_{w i}(S)$ are further described below. Constants $k_{i}$ and $k_{w i}$ represent cell death rates in fluid and wall environments, respectively, $\beta_{i}$ is the rate of sloughing of wallattached bacteria into the fluid, and $\alpha_{i}$ is the rate coefficient of adhesion to the wall surface for strain $i$. Axial and radial nutrient diffusion coefficients are given by $d_{x}^{S}, d_{r}^{S}$, respectively; axial and radial bacterial motility coefficients for the $i$ th strain are $d_{x}^{i}, d_{r}^{i}$, respectively. A model assumption is that there is a maximum attainable areal density of wall-attached bacteria $w_{\max }$ and

$$
W=\sum_{i} \frac{w^{i}}{w_{\max }}
$$

represents the occupation fraction. Danckwerts' boundary conditions describe the interface conditions between the up-stream and down-stream flow and the reactor. They are as follows :

at $x=0$ :

$$
\begin{aligned}
v(r) S^{0} & =-d_{x}^{S} S_{x}+v(r) S \\
0 & =-d_{x}^{i} u_{x}^{i}+v(r) u^{i},
\end{aligned}
$$

at $x=L$ :

$$
S_{x}=u_{x}^{i}=0
$$

The radial boundary conditions at $r=R$ are:

$$
\begin{aligned}
& 0=d_{r}^{S} S_{r}+\sum_{i} \gamma_{i}^{-1} w^{i} f_{w i}(S) \\
& 0=d_{r}^{i} u_{r}^{i}+\alpha_{i} u^{i}(1-W)-w^{i}\left[f_{w i}(S)\left(1-G_{i}(W)\right)+\beta_{i}\right]
\end{aligned}
$$

In addition, $S, u, w$ satisfy (non-negative) initial conditions at $t=0$ :

$$
\begin{aligned}
S(x, y, z, 0) & =S_{0}(x, y, z) \\
u^{i}(x, y, z, 0) & =u_{0}^{i}(x, y, z) \\
w^{i}(x, y, z, 0) & =w_{0}^{i}(x, y, z) .
\end{aligned}
$$

We assume that $S_{0}, u_{0}^{i}, w_{0}^{i}$ are continuous. 
It will also be of interest to allow the "charging" of the reactor with microbes to take place via the boundary condition at $x=0$ by replacing zero on the left side of (51) by $v(r) u^{0 i}(t)$, where $u^{0 i}(t) \equiv u^{0 i}$, a constant, on $0 \leq t \leq t_{0}$ and $u^{0 i}(t)=0, t \geq t_{0}$.

Keeping in mind that $0 \leq w^{i} \leq w_{\infty}$, the initial data and solutions $S, u^{i}, w^{i}$ must satisfying:

$$
S, u^{i}, w^{i} \geq 0, \quad \sum_{i} w^{i} \leq w_{\infty} .
$$

Hereafter, we refer to these restrictions as the range conditions.

We stress here that the model equations describe competition among the $n$ bacterial strains for limited wall colonization sites as well as for limited substrate. A strain may be a good competitor by being able to grow at low substrate concentrations and/or by a relatively strong ability for surface attachment.

For brevity, we let $L^{S}$ and $L^{i}$ denote the differential operators for the $S$ and $u^{i}$ equations so they become:

$$
\begin{gathered}
S_{t}=L^{S} S-\sum_{i} \gamma_{i}^{-1} u^{i} f_{u i}(S) \\
u_{t}^{i}=L^{i} u^{i}+u^{i}\left(f_{u i}(S)-k_{i}\right)
\end{gathered}
$$

\subsection{Eigenvalue Problems}

A family of non-standard eigenvalue problems plays a central role in our analysis so we introduce them here. The adjoint operator to $L^{S}\left(L^{i}\right)$ with homogeneous boundary conditions (51) with $S^{0}=0,(52)$, and radial boundary condition $S_{r}=0$ is denoted by $L_{S}\left(L_{i}\right) . L_{S}$ is given by:

$$
L_{S} \phi=d_{x}^{S} \phi_{x x}+d_{r}^{S}\left[\phi_{y y}+\phi_{z z}\right]+v(r) \phi_{x}
$$

with homogeneous boundary conditions

$$
\begin{array}{ll}
0=d_{x}^{S} \phi_{x}+v(r) \phi, & x=L \\
0=\phi_{x}, & x=0 \\
0=\phi_{r}, & r=R
\end{array}
$$

and similarly for $L_{i}$. It's as if the direction of flow through the reactor changed from left to right to right to left. Denote by $-\lambda^{S}$ the principal eigenvalue of the eigenvalue problem:

$$
L_{S} \phi=\lambda \phi,
$$

together with the above boundary conditions. Then $-\lambda^{S}<0$ and the corresponding eigenfunction $\phi$ satisfies $\phi>0$ on $\bar{\Omega}$ and can be normalized by assuming that it attains a maximum of unity (see Appendix 6 in [33]). Let $-\lambda^{i}<0$ be the principal eigenvalue of $L_{i}$ subject to analogous homogeneous 
boundary conditions. It is well-known that $-\lambda^{i}<0$ is also the principal eigenvalue of $L^{i}$ corresponding to boundary conditions (51),(52), and $u_{r}=0$ on $r=R$.

Another important pair of eigenvalue problems is the following.

$$
\begin{aligned}
\lambda u & =L^{i} u+a u, \quad \Omega \\
\lambda w & =b w+\alpha u, \quad r=R \\
0 & =d_{r} u_{r}+\alpha u-c w, \quad r=R \\
0 & =-d_{x} u_{x}+v(r) u, \quad x=0 \\
0 & =u_{x}, \quad x=L
\end{aligned}
$$

The corresponding adjoint problem is given by:

$$
\begin{aligned}
\lambda u & =L_{i} u+a u, \quad \Omega \\
\lambda w & =b w+c u, \quad r=R \\
0 & =d_{r} u_{r}+\alpha u-\alpha w, \quad r=R \\
0 & =d_{x} u_{x}+v(r) u, \quad x=L \\
0 & =u_{x}, \quad x=0
\end{aligned}
$$

In order to see in what sense (57) is adjoint to (56) we make the following observation.

Proposition 2. Let $u \in C^{2}(\Omega) \cap C^{1}(\bar{\Omega})$ satisfy the Danckwerts' boundary conditions at $x=0, L, \hat{u} \in C^{2}(\Omega) \cap C^{1}(\bar{\Omega})$ satisfy the adjoint Danckwerts' boundary conditions at $x=0, L, u, w$ satisfy the inhomogeneous radial boundary condition

$$
h=d_{r} u_{r}+\alpha u-c w, \quad r=R
$$

and $\hat{u}, \hat{w}$ satisfy the homogeneous adjoint radial boundary condition in (57). Then we have

$$
\begin{aligned}
& \int_{\Omega}\left(L^{i} u\right) \hat{u} d V+\int_{r=R}(b w+\alpha u) \hat{w} d A= \\
& \int_{\Omega}\left(L_{i} \hat{u}\right) u d V+\int_{r=R} h \hat{u}+w(b \hat{w}+c \hat{u}) d A
\end{aligned}
$$

If $h \equiv 0$, then we obtain the adjoint relation of (57) and (56).

The proof, given in [32], boils down to the use of Green's identities.

One of our main tools is the following result, Theorem 3.3 in [33]. It can be generalized to non-constant coefficients; see [32].

Proposition 3. Principal Eigenvalue. Let $\alpha, c>0$. Then there exists a real simple eigenvalue $\lambda^{*}>b$ of (56) satisfying:

$$
\begin{aligned}
& b+c<\lambda^{*} \leq a-\lambda_{i}, \text { if } b+c<a-\lambda_{i} \\
& b+c=\lambda^{*}, \quad \text { if } b+c=a-\lambda_{i} \\
& a-\lambda_{i}<\lambda^{*}<b+c \text {, if } b+c>a-\lambda_{i}
\end{aligned}
$$


Corresponding to eigenvalue $\lambda^{*}$ is an eigenvector $(\bar{u}, \bar{w})$ satisfying $\bar{u}>0$ in $\bar{\Omega}$ and $\bar{w}>0$ in $r=R$. If $\lambda$ is any other eigenvalue of (56) corresponding to an eigenvector $(u, w) \geq 0$, then $\lambda=\lambda^{*}$ and $(u, w)=c(\bar{u}, \bar{w})$ for some $c>0 . \bar{u}, \bar{w}$ are axially symmetric, i.e., in cylindrical coordinates $(r, \theta, x)$, $\bar{u}=\bar{u}(r, x), \bar{w}=\bar{w}(x)$.

$\lambda^{*}$ is also an eigenvalue of (57) corresponding to an eigenvector $(u, w)=$ $(\psi, \chi)$. Moreover, $(\psi, \chi)$ has the same uniqueness up to scalar multiple, positivity and symmetry properties as does $(\bar{u}, \bar{w})$.

\subsection{Estimates and Simulations}

We begin by establishing that bacterial growth is limited by the supplied substrate. Let $\left(\psi^{i}, \chi^{i}\right)$ be the principal eigenvector corresponding to the eigenvalue $\overline{\lambda_{i}}$ of (57) in the case that $a=0, b=-\beta_{i}, \alpha=\alpha_{i}, c=\beta_{i}, d_{r}=d_{r}^{i}, d_{x}=$ $d_{x}^{i}$. Normalize $\left(\psi^{i}, \chi^{i}\right)$ by requiring $\psi^{i}, \chi^{i} \leq \phi \leq 1$ with equality holding at some point for each inequality. By Proposition 3 and the fact that $b+c=0$, we have $\overline{\lambda_{i}}<0$ and, by the second of equations (57), $\psi^{i}<\chi^{i}$ on $r=R$.

Our first result represents a significant improvement over Theorem 3.1 in [33] in the case of a single species since we obtain useful bounds even when $k_{w i}=0$.

Theorem 7. The following estimates hold for solutions of (48)-(54):

$$
\limsup _{t \rightarrow \infty} S(t, x, y, z) \leq S^{0}
$$

uniformly in $(x, y, z) \in \Omega$ and

$$
\begin{aligned}
& \limsup _{t \rightarrow \infty}\left(\int_{\Omega} S \phi d V+\sum_{i} \gamma_{i}^{-1}\left[\int_{\Omega} u^{i} \psi^{i} d V+\int_{r=R} w^{i} \chi^{i} d A\right]\right) \leq \\
& \frac{2 \pi S^{0} \int_{0}^{R} r v(r) d r}{M}
\end{aligned}
$$

where

$$
M=\min _{1 \leq j \leq n}\left\{\lambda^{S},-\overline{\lambda_{j}}+k_{j},-\overline{\lambda_{j}}+k_{w j}\right\}
$$

Proof: It is easy to establish that $S \leq \tilde{S}$, where $\tilde{S}$ satisfies $S_{t}=L^{S} S$ with homogeneous radial boundary condition $S_{r}=0$ and (51),(52), by a simple comparison argument. Furthermore, noting that $S=S^{0}$ is a steady state of this comparison equation, the linearization of which having a dominant negative eigenvalue, we conclude that $\tilde{S} \rightarrow S^{0}$ as $t \rightarrow \infty$ uniformly in $(x, y, z) \in \Omega$.

Now, corresponding to the normalized eigenfunctions $\phi, \psi^{i}, \chi^{i}$, define

$$
X=\int_{\Omega} \phi S d V, Y^{i}=\int_{\Omega} \psi^{i} u^{i} d V, Z=\int_{r=R} \chi^{i} w^{i} d A .
$$


We note the following, which follow by integration by parts and Green's third identity applied to the two dimensional Laplacian in $y, z$ and using the boundary conditions satisfied by $S$ :

$$
\begin{aligned}
\int_{\Omega} \phi L^{S} S d V= & \int_{\Omega} S L_{S} \phi d V-\sum_{i} \gamma_{i}^{-1} \int_{r=R} \phi w^{i} f_{w i}(S) d A \\
& +S^{0} \int_{r \leq R} v(r) \phi(0, y, z) d y d z \\
= & -\lambda^{S} X-\sum_{i} \gamma_{i}^{-1} \int_{r=R} \phi w^{i} f_{w i}(S) d A \\
& +S^{0} \int_{r \leq R} v(r) \phi(0, y, z) d y d z
\end{aligned}
$$

Differention of $X, Y^{i}, Z^{i}$ and using these relations leads to the following:

$$
\begin{aligned}
X_{t}= & -\lambda^{S} X-\sum_{i} \gamma_{i}^{-1} \int_{\Omega} u^{i} \phi f_{u i}(S) d V-\sum_{i} \gamma_{i}^{-1} \int_{r=R} \phi w^{i} f_{w i}(S) d A \\
& +S^{0} \int_{r \leq R} v(r) \phi(0, y, z) d y d z \\
Y_{t}^{i}= & \int_{\Omega} \psi^{i} L^{i} u^{i} d V+\int_{\Omega} u^{i} \psi^{i}\left[f_{u i}(S)-k_{i}\right] d V \\
Z_{t}^{i}= & \int_{r=R} \chi^{i}\left[-\beta_{i} w^{i}+\alpha_{i} u^{i}\right] d A+\int_{r=R} \chi^{i} w^{i}\left[f_{w i}(S) G_{i}(W)-k_{w i}\right] \\
& -\chi^{i} \alpha_{i} u^{i} W d A
\end{aligned}
$$

Now, using the adjoint relation (58), the eigenvalue problem satisfied by $\left(\psi^{i}, \chi^{i}\right)$, and $\psi^{i}<\chi^{i}$ on $r=R$, we find that 


$$
\begin{aligned}
\left(Y^{i}+Z^{i}\right)_{t}= & \int_{\Omega} \psi^{i} L^{i} u^{i} d V+\int_{r=R} \chi^{i}\left[-\beta_{i} w^{i}+\alpha_{i} u^{i}\right] d A \\
& +\int_{\Omega}\left(f_{u i}(S)-k_{i}\right) \psi^{i} u^{i} d V+\int_{r=R} \chi^{i} w^{i}\left[f_{w i}(S) G_{i}(W)-k_{w i}\right] \\
& -\alpha_{i} \chi^{i} u^{i} W d A \\
= & \int_{\Omega}\left(L_{i} \psi^{i}\right) u^{i} d V+\int_{r=R} \psi^{i}\left[\alpha_{i} u^{i} W+w^{i} f_{w i}(S)\left(1-G_{i}(W)\right)\right] \\
& +w^{i}\left[-\beta_{i} \chi^{i}+\beta_{i} \psi^{i}\right] d A+\int_{\Omega} f_{u i}(S) \psi^{i} u^{i} d V \\
& +\int_{r=R} \chi^{i} w^{i} f_{w i}(S) G_{i}(W) d A-k_{i} Y^{i}-k_{w i} Z^{i} \\
& -\int_{r=R} \alpha_{i} \chi^{i} u^{i} W d A \\
= & \lambda_{i}\left(Y^{i}+Z^{i}\right)-k_{i} Y^{i}-k_{w i} Z^{i}+\int_{\Omega} f_{u i}(S) \psi^{i} u^{i} d V \\
& +\int_{r=R} w^{i} f_{w i}(S)\left[G_{i}(W) \chi^{i}+\left(1-G_{i}(W)\right) \psi^{i}\right] d A \\
& +\int_{r=R} \alpha_{i} u^{i} W\left(\psi^{i}-\chi^{i}\right) d A \\
\leq & \lambda_{i}\left(Y^{i}+Z^{i}\right)-k_{i} Y^{i}-k_{w i} Z^{i}+\int_{\Omega} f_{u i}(S) \psi^{i} u^{i} d V \\
& +\int_{r=R} w^{i} f_{w i}(S) \chi^{i} d A \\
+ & =(62) \\
& \\
& \\
&
\end{aligned}
$$

Let $Q=X+\sum_{i} \gamma_{i}^{-1}\left(Y^{i}+Z^{i}\right)$. Using $\chi^{i}, \psi^{i} \leq \phi \leq 1$, we find that

$$
\begin{aligned}
Q_{t} \leq & -\lambda^{S} X+\sum_{i} \gamma_{i}^{-1}\left(\overline{\lambda_{i}}-k_{i}\right) Y^{i}+\sum_{i} \gamma_{i}^{-1}\left(\overline{\lambda_{i}}-k_{w i}\right) Z^{i} \\
+ & \sum_{i} \int_{\Omega} \gamma_{i}^{-1} u^{i} f_{u i}(S)\left[\psi^{i}-\phi\right] d V+\sum_{i} \int_{r=R} \gamma_{i}^{-1} w^{i} f_{w i}(S)\left[\chi^{i}-\phi\right] d A \\
& +S^{0} \int_{r \leq R} v(r) \phi(0, y, z) d y d z \\
\leq & -\lambda^{S} X+\sum_{i} \gamma_{i}^{-1}\left(\overline{\lambda_{i}}-k_{i}\right) Y^{i}+\sum_{i} \gamma_{i}^{-1}\left(\overline{\lambda_{i}}-k_{w i}\right) Z^{i} \\
& +S^{0} \int_{r \leq R} v(r) \phi(0, y, z) d y d z \\
\leq & -\min _{j}\left\{\lambda^{S},-\overline{\lambda_{j}}+k_{j},-\overline{\lambda_{j}}+k_{w j}\right\} Q+2 \pi S^{0} \int_{0}^{R} r v(r) d r .
\end{aligned}
$$

Therefore,

$$
\limsup _{t \rightarrow \infty} Q(t) \leq \frac{2 \pi S^{0} \int_{0}^{R} r v(r) d r}{\min _{j}\left\{\lambda^{S},-\overline{\lambda_{j}}+k_{j},-\overline{\lambda_{j}}+k_{w j}\right\}}
$$


This completes our proof.

As the numerator of the fraction on the right side of (59) is the net flux of nutrient into the reactor across $x=0,(59)$ says precisely that the output of organisms is limited by the input of substrate. Since $\psi^{i}>0$ is continuous on $\bar{\Omega},(59)$ implies the existence of an a priori asymptotic estimate for $\int_{\Omega} u^{i} d V$ for each $i$.

The $n$-strain model is obviously less mathematically tractable than the pure culture case $n=1$ and we can say very little about its asymptotic behavior via mathematical analysis. Aside from the washout state

$$
S=S^{0}, u_{i}=0, w_{i}=0
$$

there are the mono-culture equilibria $E_{i}$ :

$$
S=S_{i}>0, u_{i}>0, w_{i}>0, u_{j}=0, w_{j}=0, j \neq i
$$

which exist when a principal eigenvalue $\lambda_{i}^{*}>0$ and a non-degeneracy condition are satisfied (see Theorem 6 ). The main interest focuses on coexistence equilibria in which one or more strains coexist. In [32] it was shown that the linearized stability of the monoculture equilibrium $E_{i}$ to invasion by strain $j$ depends on the sign of a principal eigenvalue. However, because we have yet to show that system (48)-(54) defines a well-behaved semiflow, we have been unable to exploit the stability results so as to obtain persistence of multiple strains. We expect that the inclusion of wall-attached cell motility as in section 4.1 may yield such a system.

Parameters for the simulations have been chosen following Freter et al [25], modified for the different units used here (biomass density as opposed to cell density used by Freter) following Ballyk et al [4]. The initial conditions for wall-attached bacteria and planktonic bacteria are $S^{0}=S_{0}, w_{0}^{i}=0$, and $u_{0}^{i}=10^{-6} \mathrm{~g} / \mathrm{ml}$, respectively. In order to provide a steady-state profile, the equations were integrated to time $T=35,000$ hours at which point no further change could be detected.

The other parameters are as follows. We set $d_{x}^{S}=d_{r}^{S}=0.2 \mathrm{~cm}^{2} / h r, d_{x}^{u_{i}}=$ $d_{r}^{u_{i}}=0.002 \mathrm{~cm}^{2} / h r$ for $i=1,2,3$. The concentration of the substrate feed, $S_{0}=2.09 \times 10^{-6} \mathrm{~g} / \mathrm{ml}$. The fluid in the center of the tube is $V_{\max }=5 \mathrm{~cm} / \mathrm{hr}$. The velocity is higher than suggested by the biology. However, coexistence does not seem possible at lower velocities (holding all other parameters fixed except for uptake functions, $m_{i}, a_{i}$ ). The larger velocity apparently provides a more uniform nutrient field near the tube wall allowing the microbes to persist. We use Monod uptake functions, $f_{w_{i}}(S)=f_{u_{i}}(S)=m_{i} S /\left(a_{i}+S\right)$ with $m_{1}=1.66 / \mathrm{hr}, a_{1}=9 \times 10^{-7} \mathrm{~g} / \mathrm{ml}, m_{2}=.277 / \mathrm{hr}, a_{2}=1 \times 10^{-8} \mathrm{~g} / \mathrm{ml}$, and $m_{3}=.45 / \mathrm{hr}, a_{3}=1.05 \times 10^{-7} \mathrm{~g} / \mathrm{ml}$.

Finally, the rate of adhesion is $\alpha_{i}=500 \mathrm{~cm} / \mathrm{hr}$; the maximum areal biomass density of adherent bacteria is $w_{\infty}=2.78 \times 10^{-6} \mathrm{~g} / \mathrm{cm}^{2}$; the yield constant $\gamma=0.75$. The planktonic cell and adherent cell death rates are $k_{i}=k_{w_{i}}=.01 h^{-1}$. 
Our simulations show that three populations can coexist in the flow reactor; these three populations compete for two limited resources, namely substrate and wall-attachment space (a refuge from washout). The three organisms differ only in their uptake functions $\left(m_{i}, a_{i}\right)$; death rates, wall-affinities, sloughing rates, and yield constants are identical for the three populations. Fig. 9 (top) and Fig. 11 (top) show transient oscillations in the total planktonic and wall-attached populations, respectively, of each of the three organisms prior to reaching steady state. Coexistence is facilitated by a spatially inhomogeneous substrate steady state profile as depicted in Fig. 9 (bottom); the choice of substrate uptake functions gives each competitor an advantage over its rivals in a region of the bio-reactor. Equilibrium distributions of the three planktonic populations are shown in Fig. 10; their distributions on the reactor wall are shown in Fig. 11 (bottom). The latter form a pattern of segregation: roughly, one organism dominates the nutrient-rich upstream end, a second dominates an adjacent downstream segment and a third appears to share with the second organism the far downstream end. A similar segregation profile was shown in [4] for the analogous one-space dimensional system with three populations and in [36] for an approximate hyperbolic system with two populations. Although not shown here, populations one and two coexist in the absence of population three using the same parameters and initial data as above for the first two populations.

\section{References}

1. R. Aris: Mathematical Modeling, a Chemical Engineer's Perspective (Academic Press, New York 1999)

2. J. Bailey, D. Ollis: Biochemical Engineering Fundamentals, 2nd edn (McGraw Hill, New York 1986)

3. M. Ballyk, D. Jones, D. Le, H.L. Smith: Effects of random motility on microbial growth and competition in a flow reactor, SIAM J. Appl. Math. 59, 2 (1998) pp 573-596

4. M. Ballyk, D. Jones, H.L. Smith: Microbial competition in reactors with wall attachment: a comparison of chemostat and plug flow models, Microbial Ecology 41 (2001) pp 210-221

5. M. Ballyk, H.L. Smith: A Flow reactor with wall growth. In: Mathematical Models in Medical and Health Sciences, ed by M. Horn, G. Simonett, G. Webb (Vanderbilt University Press, Nashville, TN 1998)

6. M. Ballyk, H.L. Smith: A model of microbial growth in a plug flow reactor with wall attachment, Mathematical Biosciences 158 (1999) pp 95-126

7. B. Baltzis, A. Fredrickson: Competition of two microbial populations for a single resource in a chemostat when one of them exhibits wall attachment, Biotechnology and Bioengineering 25 (1983) pp 2419-2439

8. R. Bakke, M.G. Trulear, J.A. Robinson, W.G. Characklis: Activity of Pseudomonas aeruginosa in Biofilms: Steady State, Biotechnology and Bioengineering 26 (1984) pp 1418-1424 
9. H. Berg: Random Walks in Biology (Princeton University Press, Princeton, NJ 1983)

10. A. Berman, R. Plemmons: Nonnegative Matrices in the Mathematical Sciences (Academic Press, New York 1979)

11. J. Bryers, ed: Biofilms II, Process Analysis and Applications, Wiley series in Ecol. and Appl. Microbiology (Wiley-Liss, NY 2000)

12. W. Characklis, K. Marshall (Eds): Biofilms, Wiley Series in Ecological and Applied Microbiology (John Wiley \& Sons, New York 1990)

13. W. Characklis, M. Trulear, N. Stathopoulus, L. Chang: Oxidation and destruction of microbial films. In: Water Chlorination: Environmental Impact and Health, vol.3, ed by Jolley, Brungs, Cummings (Ann Arbor Science, Ann Arbor, MI, 1980) pp 349

14. and J.P. Keener: the Role of the biofilm matrix in structural deveopment, Mathematical Medicine and Biology 21 (2004) 147-166

15. N.G. Cogan: Effects of persister formation on bacterial response to dosing, Journal of Theoretical Biology 238, Issue 3, 7 February (2006) 694-703

16. J. Costerton: Overview of microbial biofilms, J. Indust. Microbiol. 15 (1995) pp $137-140$

17. J. Costerton, P. Stewart, E. Greenberg: Bacterial Biofilms: A common cause of persistent infections, Science 284 (1999) pp 1318-1322

18. J. Costerton, Z. Lewandowski, D. Debeer, D. Caldwell, D. Korber, G. James: Biofilms, The customized microniche, Journal of Bacteriology 176 (1994) pp 2137-2142

19. J. Costerton, Z. Lewandowski, D. Caldwell, D. Korber, H. Lappin-Scott: Microbial biofilms, Annual Review of Microbiology 49 (1995) pp 711-745

20. O. Diekmann, J. Heesterbeek: Mathematical Epidemiology of Infectious Diseases, Model Building, Analysis and Interpretation (Wiley, Chichester 2000)

21. R. Dillon, L. Fauci, A. Fogelson, D. Gaver: Modeling biofilm processes using the immersed boundary method, J. Computational Physics 129 (1996) pp 57-73

22. J. Dockery, I. Klapper: Finger formation in biofilm layers, SIAM J. Appl. Math. 62 (2001) pp 853-869

23. H.J. Eberl, D.F. Parker, M.C.M. van Loosdrecht: A new Deterministic SpatioTemporal Continuum Model for Biofilm Development, J. Theoretical Medicine 3(3) (2001) 161-175

24. R. Freter: Interdependence of mechanisms that control bacterial colonization of the large intestine, Microecology and Therapy 14 (1984) pp 89-96

25. R. Freter: Mechanisms that control the microflora in the large intestine. In: Human Intestinal Microflora in Health and Disease, ed by D. Hentges (Academic Press, New York 1983)

26. R. Freter, H. Brickner, J. Fekete, M. Vickerman, K. Carey: Survival and implantation of Escherichia coli in the intestinal tract, Infection and Immunity 39 (1983) pp 686-703

27. R. Freter, H. Brickner, S. Temme: An understanding of colonization resistance of the mammalian large intestine requires mathematical analysis, Microecology and Therapy 16 (1986) pp 147-155

28. C.A. Fux, J.W. Costerton, P.S. Stewart, and P. Stoodley, Survival strategies of infectious biofilms, Trends in Microbiology, 13 January (2005).

29. D. Herbert, R. Elsworth, R. Telling: The continuous culture of bacteria; a theoretical and experimental study, J. of Canadian Microbiology 14 (1956) pp $601-622$ 
30. M. Imran, D. Jones, H.L. Smith: Biofilms and the plasmid maintenance question, Math. Biosci. 193 (2005) pp 183-204

31. M. Imran, H.L. Smith: A Mathematical Model of Gene Transfer in a Biofilm, to appear as a chapter in, Vol.1: Mathematics for Ecology and Environmental Sciences, Springer (2006).

32. D. Jones, H. Kojouharov, D. Le, H.L. Smith: Bacterial wall attachment in a flow reactor: mixed culture, Canadian Applied Math. Quarterly 10 (2004) pp $111-138$

33. D. Jones, H. Kojouharov, D. Le, H.L. Smith: Bacterial wall attachment in a flow reactor, SIAM J. Appl. Math. 62 (2002) pp 1728-1771

34. D. Jones, H. Kojouharov, D. Le, H.L. Smith: Microbial Competition for Nutrient in a $3 D$ Flow Reactor, Dynamics of Continuous, Discrete and Impulsive Dynamical Systems 10 (2003) pp 57-67

35. D. Jones, H. Kojouharov, D. Le, H.L. Smith: The Freter model: a simple model of biofilm formation, J. Math. Biology 47 (2003) pp 137-152

36. D. Jones, H.L. Smith: Microbial competition for nutrient and wall sites in plug flow, SIAM J. Applied Math. 60 (2000) pp 1576-1600

37. M. Klausen, A. Heydorn, P. Ragas, L. Lambertsen, A. Aaes-Jorgensen, S. Molin, T. Tolker-Nielsen: Biofilm formation by Pseudomonas aeruginosa wild type, flagella and type IV pili mutants, Molecular Microbiology 48 (2003) pp 15111524

38. J.-U. Kreft, C. Picioreanu, J. Wimpenny, M. van Loosdrecht: Individual-based modelling of biofilms, Microbiology 147 (2001) pp 2897-2912

39. C.M. Kung, B. Baltzis: The growth of pure and simple microbial competitors in a moving distributed medium, Math. Biosci. 111 (1992) pp 295-313

40. C.S.Laspidou and B.E. Rittmann: Modeling the development of biofilm density including active bacteria, inert biomass, and extracellular polymeric substances, Water Research 38 (2004) pp 3349-3361

41. D. Noguera, S. Okabe, C. Picioreanu: Biofilm Modeling: Present Status and Future Directions, Water Science Technology 39 (1999) pp 273-278

42. D. Noguera, G. Pizarro, D. Stahl, B. Rittman, Simulation of multispecies biofilm development in three dimensions, Wat.Sci. Tech. 39 (1999) 123-130

43. G. O'Toole and R. Kolter: Flagellar and twitching motility are necessary for Pseudomonas aeruginosa biofilm development, Molecular Microbiol. 30 (1998) pp 295-304

44. C. Picioreanu, M.C.M. van Loosdrecht, J. Heijnen: Mathematical Modeling of biofilm structure with a hybrid differential-discrete cellular automaton approach, Biotechnol. Bioeng. 58 (1998) pp 101-116

45. S. Pilyugin and P. Waltman: The simple chemostat with wall growth, SIAM J. Appl. Math. 59 (1999) pp 1552-1572

46. L. Pratt and R. Kolter: Genetic analysis of Escherichia coli biofilm formation: roles of flagella, motility, chemotaxis and type I pili, Molecular Microbiol. 30 (1998) pp 285-293

47. A. Reisner, J. Haagensen, M. Schembri, E. Zechner, S. Molin: Developement and maturation of Escherichia coli K-12 biofilms, Molecular Microbiology 48 (2003) pp 933-946

48. H.L. Smith: A semilinear hyperbolic system, Proceedings of the Mathematics Conference, ed by S. Elyadi, F. Allan, A. Elkhader, T. Muhgrabi, M. Saleh (World Scientific 2000) 
49. H.L. Smith, P. Waltman: The Theory of the Chemostat (Cambridge University Press, New York 1995)

50. H.L. Smith, X.-Q. Zhao: Microbial growth in a plug flow reactor with wall attachment and cell motility, JMAA 241 (2000) pp 134-155

51. G. Stephanopoulus, G. Lapidus: Chemostat dynamics of plasmid-bearing plasmid-free mixed recombinant cultures, Chem. Engng. Sci. 43 (1988) pp 49-57

52. E. Stemmons, H.L. Smith, H.L.: Competition in a chemostat with wall attachment, SIAM J. Appl.Math. 61 (2000) pp 567-595

53. P. Stewart, G. Mcfeters, C.-T. Huang: Biofilm control by antimicrobial agents, Chapter 11. In: Biofilms II: Process Analysis and Applications, ed by J. Bryers (Wiley-Liss 2000)

54. S.M. Hunt, M.A. Hamilton, and P.S. Stewart: A 3D model of antimicrobial action on biofilms, Water Science and Technology 52 (7) (2005) 143-148.

55. H. Topiwala and G. Hamer: Effect of wall growth in steady-state continuous cultures, Biotechnol. Bioeng. 13 (1971) pp 919-922

56. C.Y. Wen, L.T. Fan: Models for flow systems and chemical reactors. In: Chemical Processing and Engineering, vol. 3 (Marcel Dekker, New York 1975)

57. J.W.T. Wimpenny, R. Colasanti: A unifying hypothesis for the structure of microbial biofilms based on cellular automaton models, FEMS Microb. Ecol. 22 (1997) pp 1-16 


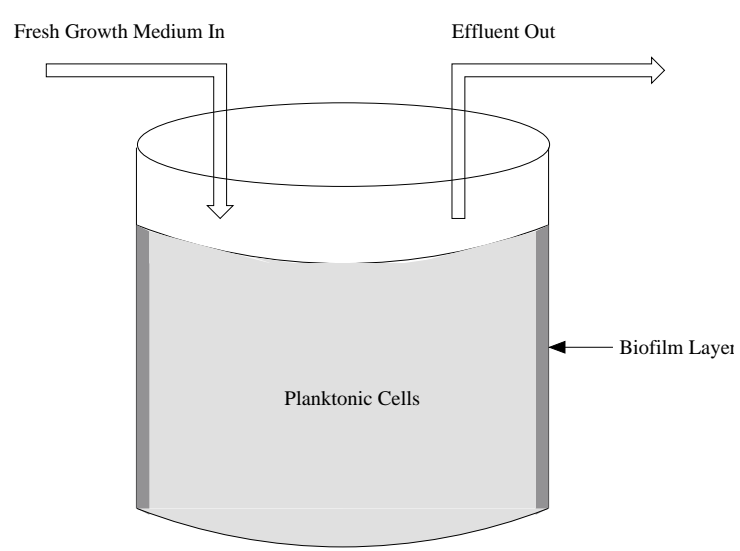

Fig. 1. Wall growth in the chemostat. Planktonic cells reside in well-mixed fluid; wall-adherent cells reside in the biofilm layer.

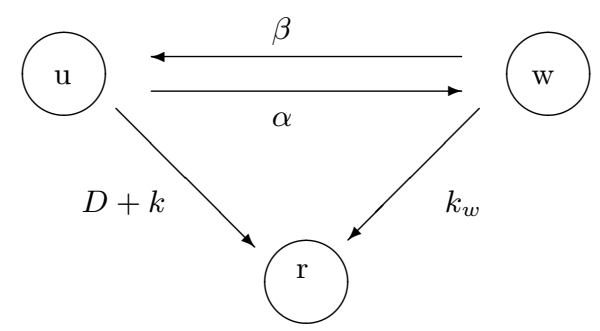

Fig. 2. Continuous-time Markov chain with absorbing state $r=$ removal by washout or death 

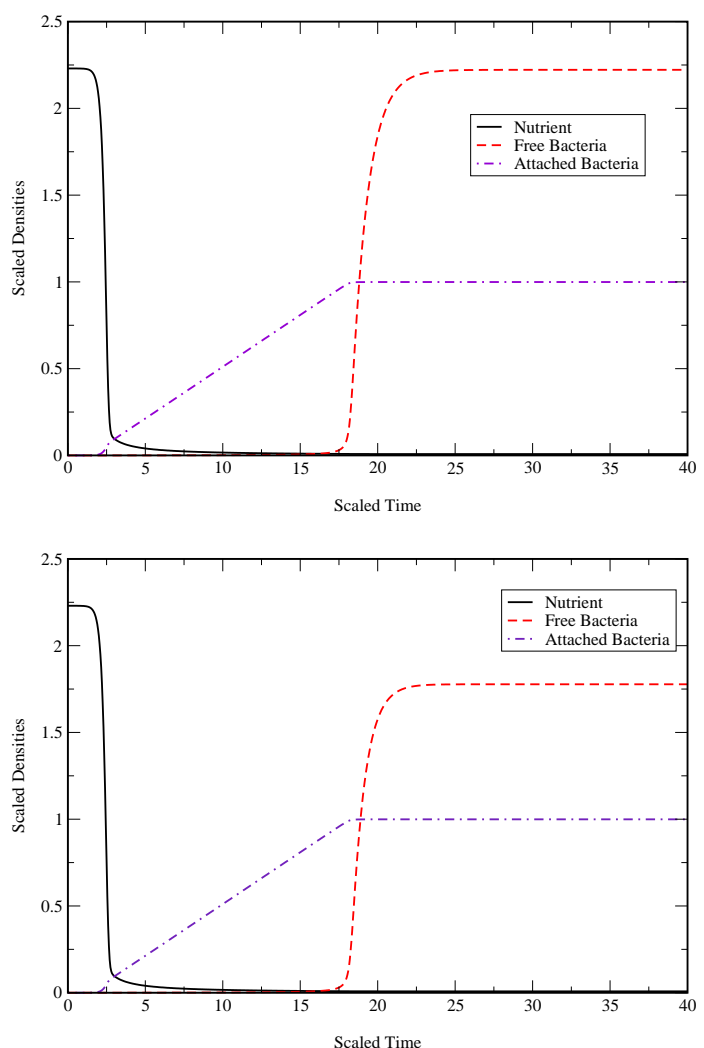

Fig. 3. Top: Biofilm formation in CSTR. Bottom: Biocide raises planktonic cell death rate

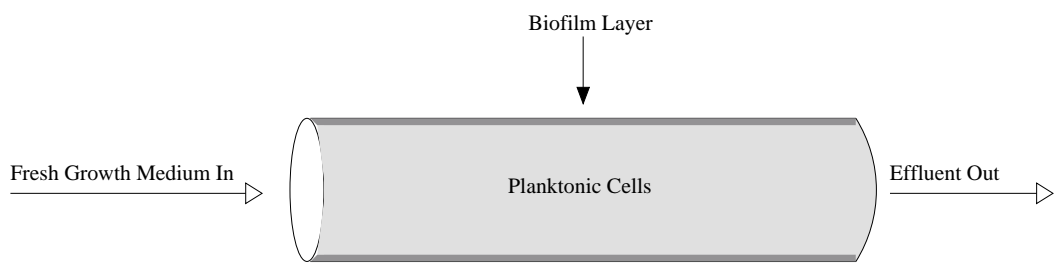

Fig. 4. Flow Reactor with biofilm 
The Biofilm Model of Freter: a review

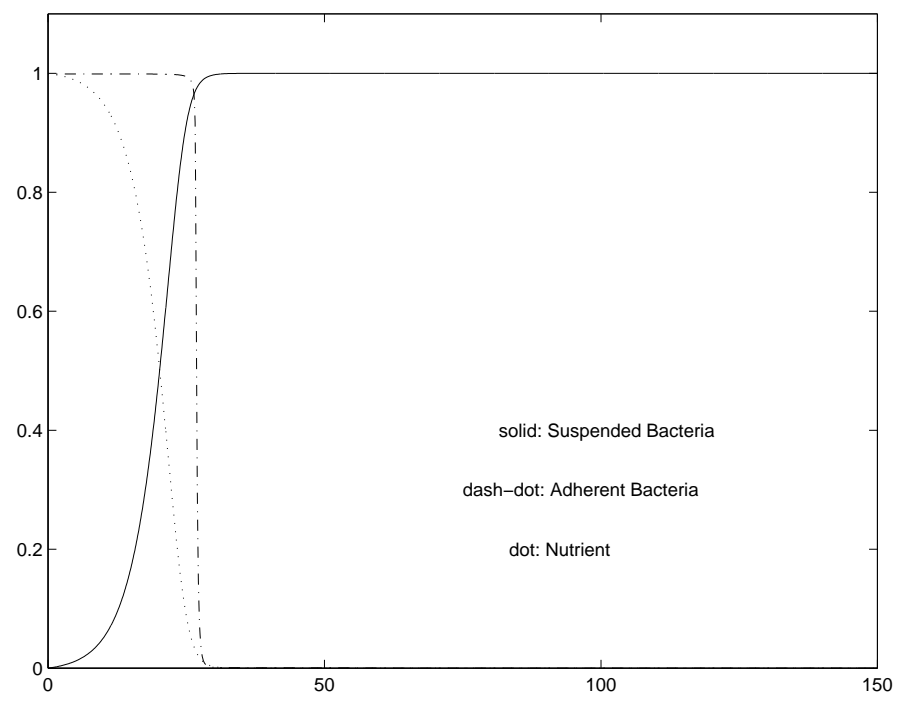

Fig. 5. Steady state solution of (17)-(19) with $k=k_{w}=0.0$

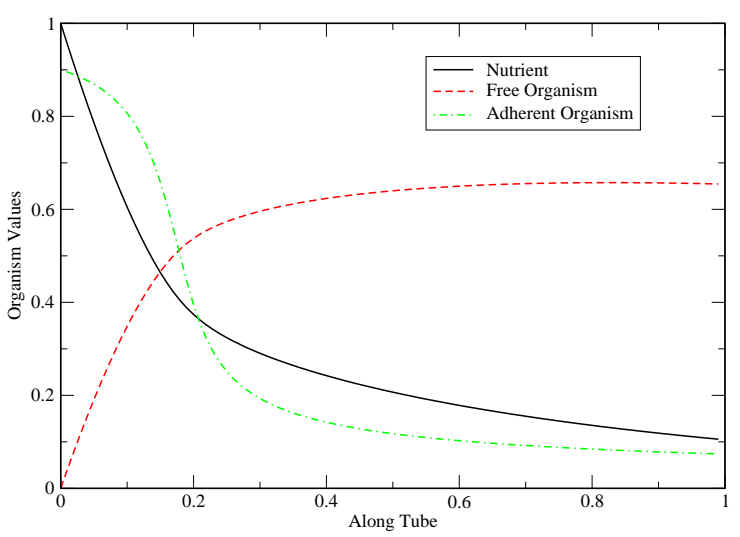

Fig. 6. Steady-state solution $(\bar{S}, \bar{u}, \bar{w})$ of (25)-(27) 

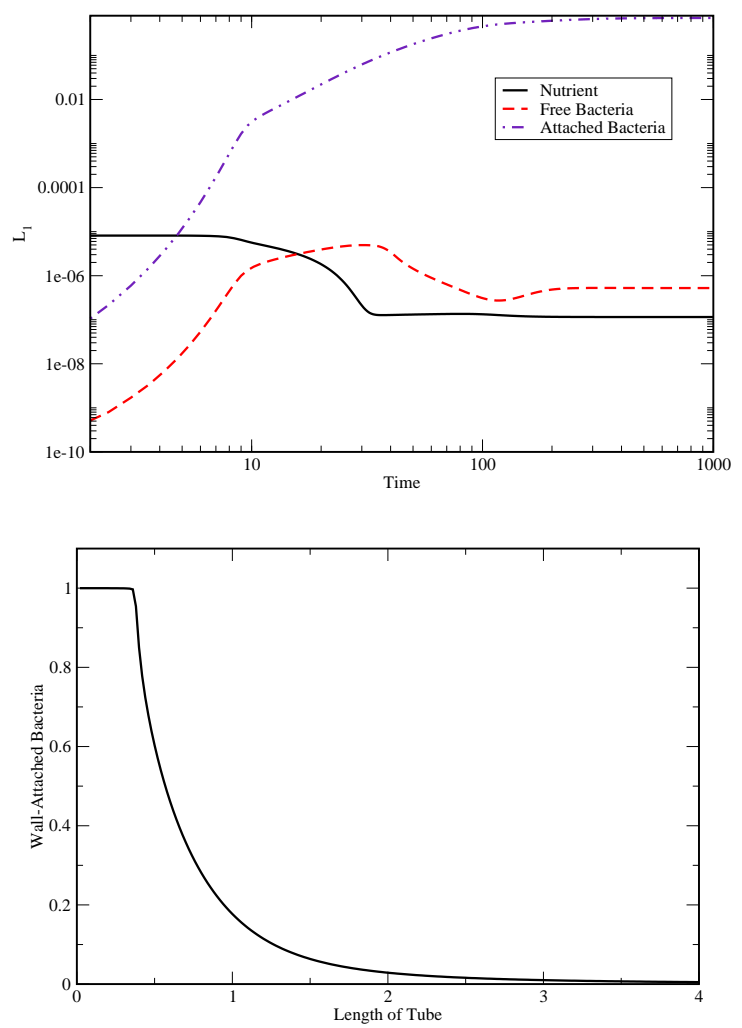

Fig. 7. Top: Time series plot of the total nutrient, free bacteria, and attached bacteria for (35)-(40). Bottom: Graph of steady-state, wall-attached bacteria 

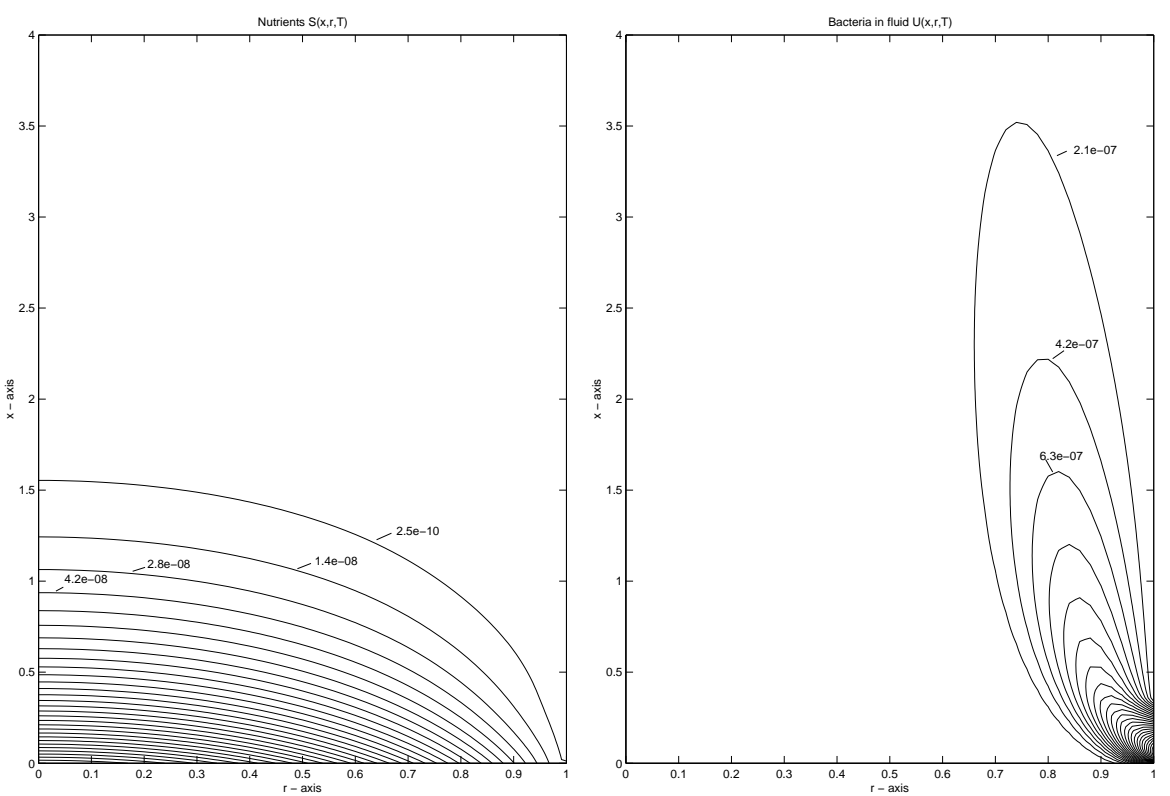

Fig. 8. Left: Contour plots of nutrient density at steady-state, ranging from $2.5 \times$ $10^{-10} \mathrm{~g} / \mathrm{ml}$ at top to $4.13 \times 10^{-7} \mathrm{~g} / \mathrm{ml}$ at bottom. Right: Contour plots of planktonic biomass density at steady state, ranging from near zero to $5.2 \times 10^{-6} \mathrm{~g} / \mathrm{ml}$ 

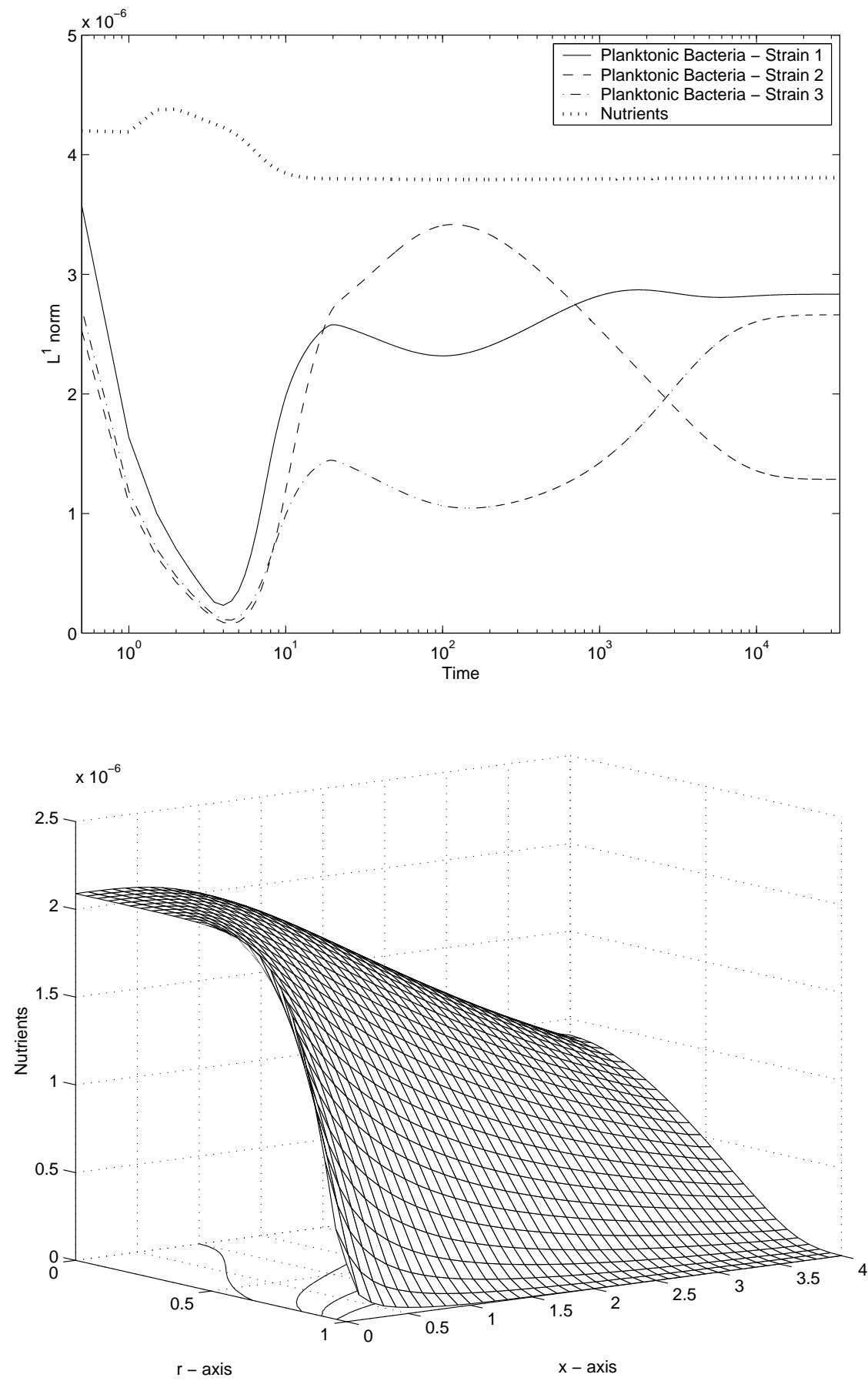

Fig. 9. $L^{1}$ norm versus time of the nutrients and free bacteria (top) and a surface plot of the nutrient density $S$ (bottom) 

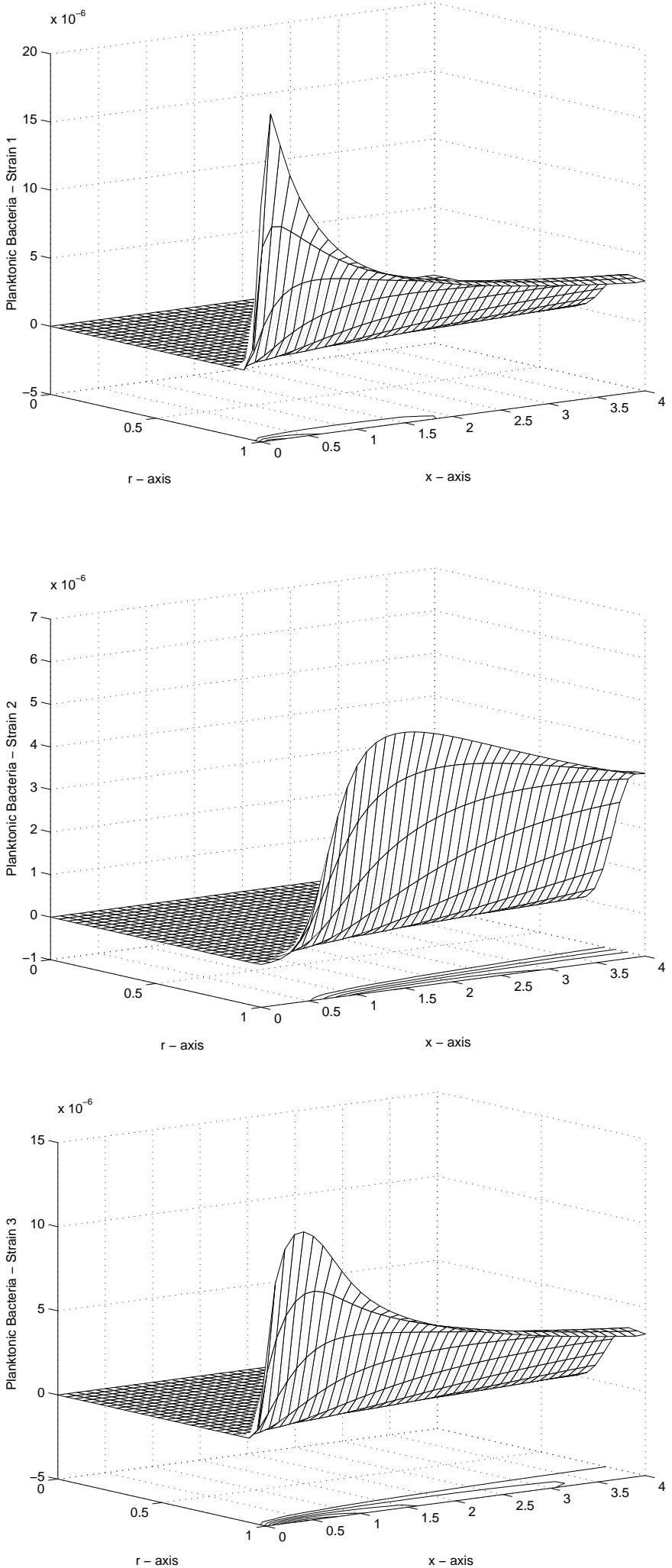

Fig. 10. Surface plots of the planktonic biomass density $u^{i}$ - strain 1 (top), strain 2 (center), strain 3 (bottom) 

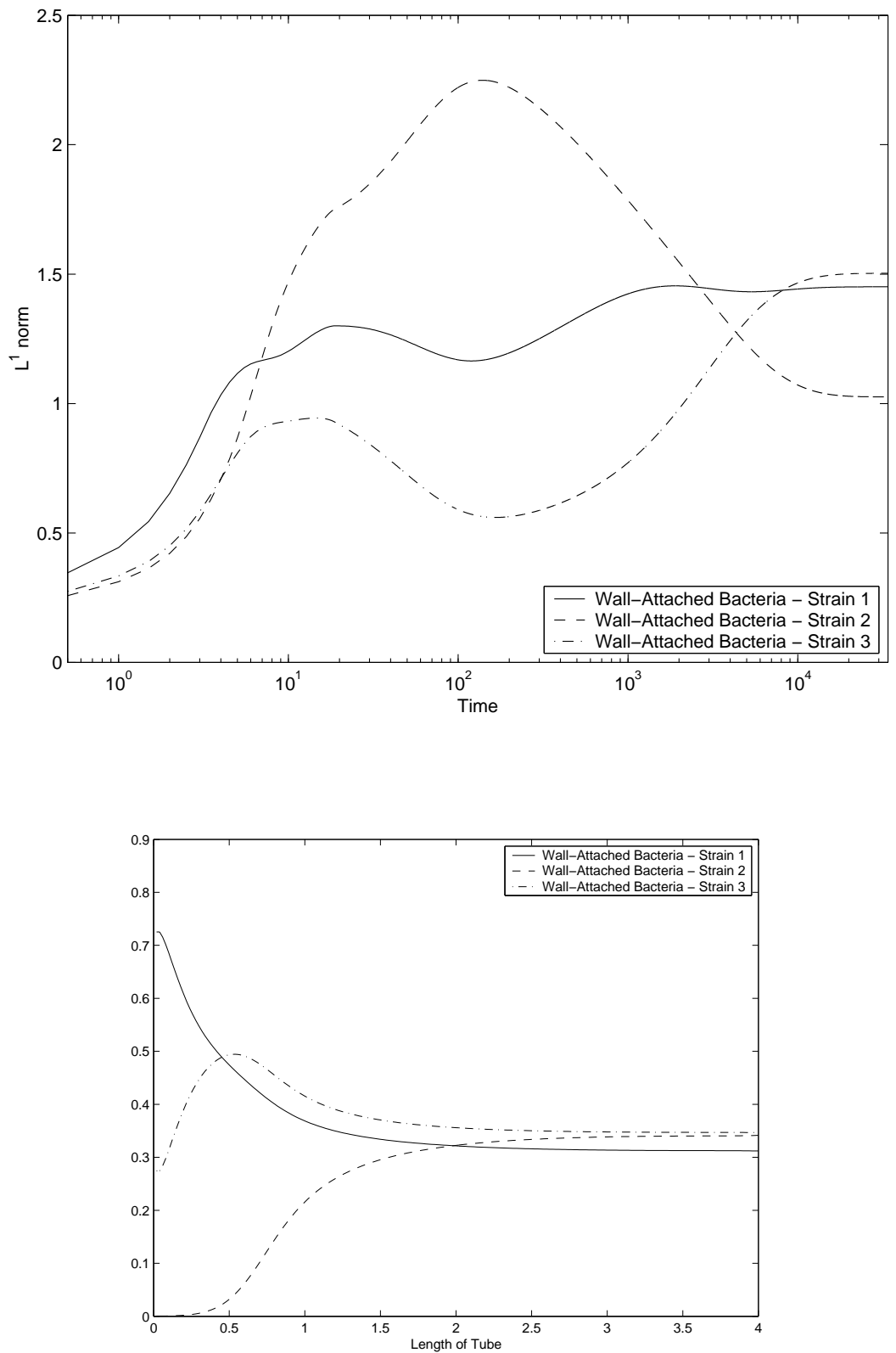

Fig. 11. $L^{1}$ norm versus time of the wall-attached bacteria (top) and a plot of the areal density of wall-attached bacteria $w^{i}$ at a steady-state (bottom) 